University of Wollongong

Research Online

Faculty of Informatics - Papers (Archive)

Faculty of Engineering and Information

Sciences

$1-1-2003$

\title{
Affine Hecke algebras, cyclotomic Hecke algebras and Clifford theory
}

Arun Ram

University of Wisconsin - Madison

Jacqueline Ramagge

University of Newcastle, ramagge@uow.edu.au

Follow this and additional works at: https://ro.uow.edu.au/infopapers

Part of the Physical Sciences and Mathematics Commons

\section{Recommended Citation}

Ram, Arun and Ramagge, Jacqueline: Affine Hecke algebras, cyclotomic Hecke algebras and Clifford theory $2003,428-466$.

https://ro.uow.edu.au/infopapers/1231

Research Online is the open access institutional repository for the University of Wollongong. For further information contact the UOW Library: research-pubs@uow.edu.au 


\title{
Affine Hecke algebras, cyclotomic Hecke algebras and Clifford theory
}

\begin{abstract}
We show that the Young tableaux theory and constructions of the irreducible representations of the Weyl groups of type A, B and D, Iwahori-Hecke algebras of types $A, B$, and $D$, the complex reflection groups $G(r$, $\mathrm{p}, \mathrm{n}$ ) and the corresponding cyclotomic Hecke algebras $\mathrm{Hr}, \mathrm{p}, \mathrm{n}$, can be obtained, in all cases, from the affine Hecke algebra of type A. The Young tableaux theory was extended to affine Hecke algebras (of general Lie type) in recent work of A. Ram. We also show how (in general Lie type) the representations of general affine Hecke algebras can be constructed from the representations of simply connected affine Hecke algebras by using an extended form of Clifford theory. This extension of Clifford theory is given in the Appendix.
\end{abstract}

Keywords

theory, algebras, hecke, clifford

Disciplines

Physical Sciences and Mathematics

Publication Details

Ram, A. \& Ramagge, J. (2003). Affine Hecke algebras, cyclotomic Hecke algebras and Clifford theory. In V. Lakshmibai, V. Balaji, V. B. Mehta, K. R. Nagarajan, K. Paranjape, P. Sankaran \& R. Sridharan (Eds.), A Tribute to C.S. Seshadri: A Collection of Articles on Geometry and Representation Theory (Trends in Mathematics) (pp. 428-466). Basel: Birkhauser Verlag. 


\title{
Affine Hecke algebras, cyclotomic Hecke algebras and Clifford theory
}

\author{
Arun Ram * \\ Department of Mathematics \\ University of Wisconsin, Madison \\ Madison, WI 53706 USA \\ ram@math.wisc.edu \\ Jacqui Ramagge \\ Department of Mathematics \\ University of Newcastle \\ NSW 2308 Australia \\ jacqui@maths.newcastle.edu .au
}

Dedicated to Professor C.S. Seshadri on the occasion of his 70th birthday

Abstract. We show that the Young tableaux theory and constructions of the irreducible representations of the Weyl groups of type A, B and D, Iwahori-Hecke algebras of types A, B, and D, the complex reflection groups $G(r, p, n)$ and the corresponding cyclotomic Hecke algebras $H_{r, p, n}$, can be obtained, in all cases, from the affine Hecke algebra of type A. The Young tableaux theory was extended to affine Hecke algebras (of general Lie type) in recent work of A. Ram. We also show how (in general Lie type) the representations of general affine Hecke algebras can be constructed from the representations of simply connected affine Hecke algebras by using an extended form of Clifford theory. This extension of Clifford theory is given in the Appendix.

\section{INTRODUCTION}

Recent work of A. Ram [Ra2,5] gives a straightforward combinatorial construction of the simple calibrated modules of affine Hecke algebras (of general Lie type as well as type A). The first aim of this paper is to show that Young's seminormal construction and all of its previously known generalizations are special cases of the construction in [Ra5]. In particular, the representation theory of

(a) Weyl groups of types A, B, and D,

(b) Iwahori-Hecke algebras of types A, B, and D,

(c) the complex reflection groups $G(r, p, n)$, and

(d) the cyclotomic Hecke algebras $H_{r, p, n}$,

can be derived entirely from the representation theory of affine Hecke algebras of type A. Furthermore, the relationship between the affine Hecke algebra and the objects in (a)-(d) always produces a natural set of Jucys-Murphy type elements and can be used to prove the standard Jucys-Murphy type theorems. In particular, we are able to use Bernstein's results about the center of the affine Hecke algebra to show that, in the semisimple case, the center of the cyclotomic Hecke algebra $H_{r, 1, n}$ is the set of symmetric polynomials in the Jucys-Murphy elements.

* Research supported in part by NSF grants DMS-9622985 and DMS-9971099 and the National Security Agency. 
A. Young's seminormal construction of the irreducible representations of the symmetric group dates from 1931 [Yg1]. Young himself generalized his tableaux to treat the representation theory of Weyl groups of types B and D [Yg2]. In 1974 P.N. Hoefsmit [Hf] generalized the seminormal construction to Iwahori-Hecke algebras of types A, B, and D. Hoefsmit's work has never been published and, in 1985, Dipper and James [DJ, Theorem 4.9] and H. Wenzl [Wz] independently treated the seminormal construction for irreducible representations for Iwahori-Hecke algebras of type A. In 1994 Ariki and Koike [AK] introduced (some of) the cyclotomic Hecke algebras and generalized Hoefsmit's construction to these algebras. The construction was generalized to a larger class of cyclotomic Hecke algebras in [Ar2]. For a summary of this work see [Ra1] and [HR].

General affine Hecke algebras are naturally associated to a reductive algebraic group and the size of the commutative part of the affine Hecke algebra depends on the structure of the corresponding algebraic group (simply connected, adjoint, etc.). The second aim of this paper is to show that it is sufficient to understand the representation theory of the affine Hecke algebra in the simply connected case. We describe explicitly how the representation theory of the other cases is derived from the simply connected case.

The machine which allows us to accomplish this reduction is a form of Clifford theory. Precisely, if $R$ is an algebra and $G$ is a finite group acting on $R$ by automorphisms then the representation theory of the ring $R^{G}$ of fixed points of the $G$-action can be derived from the representation theory of $R$ and subgroups of $G$. This is an extension of the approach to Clifford theory given by Macdonald [Mac2].

Let us state precisely what is new in this paper. The main result has three parts:

(1) The Hecke algebras $H_{r, p, n}$ of the complex reflection groups $G(r, p, n)$ can be obtained as fixed point subalgebras of the Hecke algebra $H_{r, 1, n}$ associated to the complex reflection group $G_{r, 1, n}$ via

$$
H_{r, p, n}=\left(H_{r, 1, n}\right)^{\mathbb{Z} / p \mathbb{Z}} .
$$

(2) The Hecke algebras $\tilde{H}_{L}$ of nonadjoint $p$-adic groups can be obtained as fixed point subalgebras of the Hecke algebra $\tilde{H}_{P}$ associated to the corresponding adjoint $p$-adic group, via

$$
\tilde{H}_{L}=\left(\tilde{H}_{P}\right)^{P / L} .
$$

(3) There is a form of Clifford theory (to our knowledge new) that allows one to completely determine the representation theory of a fixed point subalgebra $R^{G}$ in terms of the representation theory of the algebra $R$ and the group $G$.

We use this method to work out the representation theory of the algebras $H_{r, p, n}$ in detail. Additional results include,

(4) The discovery of the "right" affine braid groups $\mathcal{B}_{\infty, p, n}$ and affine Hecke algebras $H_{\infty, p, n}$ to associate to the cyclotomic Hecke algebras $H_{r, p, n}$. The representation theory of these new groups and algebras is completely determined from the representation theory of the classical affine braid groups $B_{\infty, 1, n}$ and the classical affine Hecke algebras $H_{\infty, 1, n}$ of type $A$ as a consequence of the results in (3).

Finally,

(5) We show how the classical trick (due to Cherednik) for determining the representation theory of the algebras $H_{r, 1, n}$ from that of $H_{\infty, 1, n}$ arises from a map from the affine Hecke algebra of type $C$ to the finite Hecke algebra of type $C$ which corresponds to a folding of the Dynkin diagram. This explanation is new. We show that such maps from the affine Hecke algebra to the finite Hecke algebra cannot exist in general type, and we work out the details of the cases where such homomorphisms do arise from foldings. 
In a recent paper Reeder [Re] has used our results to prove the Langlands classification of irreducible representations for general affine Hecke algebras. (Previously, this was known only in the simply connected case, see Kazhdan and Lusztig [KL].) This also provides a classification of the irreducible constituents of unramified principal series representations of general split reductive $p$-adic groups. (The Kazhdan-Lusztig result provides this classification for groups with connected center.)

Acknowledgements. A. Ram thanks P. Deligne for his encouragement, stimulating questions and helpful comments on the results in this paper. We thank D. Passman for providing some useful references about Clifford theory. We are grateful for the generous support of this research by the National Science Foundation, the National Security Agency and the Australian Research Council.

\section{Algebras with Young tableaux theories}

A. Young invented the theory of standard Young tableaux in order to describe the representation theory of the symmetric group $S_{n}$; the group of $n \times n$ matrices such that

(a) the entries are either 0 or 1 ,

(b) there is exactly one nonzero entry in each row and each column.

Young himself began to generalize the theory and in [Yg1] he provided a theory for the Weyl groups of type B, i.e. the hyperoctahedral groups $W B_{n} \cong(\mathbb{Z} / 2 \mathbb{Z})$ 乙 $S_{n}$ of $n \times n$ matrices such that

(a) the entries are either 0 or \pm 1 ,

(b) there is exactly one nonzero entry in each row and each column.

In the same paper Young also treated the Weyl group $W D_{n}$ of $n \times n$ matrices such that

(a) the entries are either 0 or \pm 1 ,

(b) there is exactly one nonzero entry in each row and each column,

(c) the product of the nonzero entries is 1 .

W. Specht [Sp] generalized the theory to cover the complex reflection groups $G(r, 1, n) \cong(\mathbb{Z} / r \mathbb{Z}) 2 S_{n}$ consisting of $n \times n$ matrices such that

(a) the entries are either 0 or $r$ th roots of unity,

(b) there is exactly one nonzero entry in each row and each column.

In the classification [ST] of finite groups generated by complex reflections there is a single infinite family of groups $G(r, p, n)$ and exactly 34 others, the "exceptional" complex reflection groups. The groups $G(r, p, n)$ are the groups of $n \times n$ matrices such that

(a) the entries are either 0 or $r$ th roots of unity,

(b) there is exactly one nonzero entry in each row and each column,

(c) the $(r / p)$ th power of the product of the nonzero entries is 1 .

Though we do not know of an early reference which generalizes the theory of Young tableaux to these groups, it is not difficult to see that the method that Young uses for the Weyl groups $W D_{n}$ extends easily to handle the groups $G(r, p, n)$.

Special cases of the groups $G(r, p, n)$ are

(a) $G(1,1, n)=S_{n}$, the symmetric group,

(b) $G(2,1, n)=W B_{n}$, the hyperoctahedral group (i.e. the Weyl group of type $B_{n}$ ),

(c) $G(2,2, n)=W D_{n}$, the Weyl group of type $D_{n}$, 
(d) $G(r, 1, n) \cong(\mathbb{Z} / r \mathbb{Z})<S_{n}=(\mathbb{Z} / r \mathbb{Z})^{n} \rtimes S_{n}$.

The order of $G(r, 1, n)$ is $r^{n} n$ !. Let $E_{i j}$ be the $n \times n$ matrix with 1 in the $(i, j)$ position and all other entries 0 . Then $G(r, 1, n)$ can be presented by generators

$$
s_{1}=\zeta E_{11}+\sum_{j \neq 1} E_{i i}, \quad \text { and } \quad s_{i}=E_{i, i+1}+E_{i+1, i}+\sum_{j \neq i, i+1} E_{j j}, \quad 2 \leq i \leq n,
$$

where $\zeta$ is a primitive $r$ th root of unity, and relations
(B1) $s_{i} s_{j}=s_{j} s_{i}$
if $|i-j|>1$,
(B2) $s_{i} s_{i+1} s_{i}=s_{i+1} s_{i} s_{i+1}$,
for $2 \leq i \leq n-1$,
(BB) $s_{1} s_{2} s_{1} s_{2}=s_{2} s_{1} s_{2} s_{1}$,
(C) $s_{1}^{r}=1$,
(R) $s_{i}^{2}=1$,
for $2 \leq i \leq n$.

The group $G(r, p, n)$ is the subgroup of index $p$ in $G(r, 1, n)$ generated by

$$
a_{0}=s_{1}^{p}, \quad a_{1}=s_{1} s_{2} s_{1}, \quad a_{i}=s_{i}, \quad 2 \leq i \leq n .
$$

(1.1) Cyclotomic Hecke algebras $H_{r, 1, n}$. More recently there has been an interest in IwahoriHecke algebras associated to reflection groups and there has been significant work generalizing the constructions of A. Young to these algebras. Iwahori-Hecke algebras of types A, B and D were handled by Hoefsmit [Ho] and other aspects of the theory for these algebras were developed by Dipper, James and Murphy [DJ, DJM], Gyoja [Gy] and Wenzl [Wz]. In 1994, Ariki and Koike [AK] introduced cyclotomic Hecke algebras $H_{r, 1, n}$ for the complex reflection groups $G(r, 1, n)$ and they generalized the Young tableau theory to these algebras. Theorem 3.18 below shows that the theory of $[\mathrm{AK}]$ is a special case of an even more general theory for affine Hecke algebras.

Let $u_{1}, \ldots, u_{r}, q \in \mathbb{C}, q \neq 0$. The cyclotomic Hecke algebra $H_{r, 1, n}\left(u_{1}, \ldots, u_{r} ; q\right)$ is the algebra over $\mathbb{C}$ given by generators $T_{1}, \ldots, T_{n}$ and relations
(B1) $T_{i} T_{j}=T_{j} T_{i}$,
if $|i-j|>1$,
(B2) $T_{i} T_{i+1} T_{i}=T_{i+1} T_{i} T_{i+1}$
for $2 \leq i \leq n-1$,
(BB) $T_{1} T_{2} T_{1} T_{2}=T_{2} T_{1} T_{2} T_{1}$,
(qC) $\left(T_{1}-u_{1}\right)\left(T_{1}-u_{2}\right) \cdots\left(T_{1}-u_{r}\right)=0$,
$(\mathrm{qR})\left(T_{i}-q\right)\left(T_{i}+q^{-1}\right)=0$,
for $2 \leq i \leq n$.

The algebra $H_{r, 1, n}\left(u_{1}, \ldots, u_{r} ; q\right)$ is of dimension $r^{n} n$ ! (see $[\mathrm{AK}]$ ).

(a) $H_{1,1, n}(1 ; q)$ is the Iwahori-Hecke algebra of type $A_{n-1}$.

(b) $H_{2,1, n}\left(q,-q^{-1} ; q\right)$ is the Iwahori-Hecke algebra of type $B_{n}$.

(c) If $\zeta$ is a primitive $r$ th root of 1 then $H_{r, 1, n}\left(1, \zeta, \ldots, \zeta^{r-1} ; 1\right)$ is the group algebra $\mathbb{C} G(r, 1, n)$.

Fact (c) says that the representation theory of the groups $G(r, 1, n)$ is a special case of the representation theory of the algebras $H_{r, 1, n}$.

(1.2) Cyclotomic Hecke algebras $H_{r, p, n}$. The work of Broué, Malle and Michel [BMM] demonstrated that there are cyclotomic Hecke algebras associated to most complex reflection groups (even exceptional complex reflection groups). In particular, there are cyclotomic Hecke algebras $H_{r, p, n}$ corresponding to all the groups $G(r, p, n)$ and Ariki [Ar2] has generalized the Young tableau mechanism to these groups (see also [HR]). Theorems 3.15 and the mechanism of Theorem 2.8 show that the theory of Ariki is a special case of a general construction for affine Hecke algebras. 
Let $r, p, n \in \mathbb{Z}_{>0}$ be such that $p$ divides $r$ and let $d=r / p$. Let $x_{0}, \ldots, x_{d-1} \in \mathbb{C}$ and let $\xi$ be a primitive $p$ th root of 1 . For $1 \leq j \leq r$, define

$$
u_{j}=\xi^{k} x_{\ell}, \quad \text { if } j-1=\ell p+k, \quad(0 \leq k \leq p-1,0 \leq \ell \leq d-1)
$$

i.e., $u_{1}, \ldots, u_{r} \in \mathbb{C}$ are chosen so that

$$
\left(T_{1}-u_{1}\right)\left(T_{1}-u_{2}\right) \cdots\left(T_{1}-u_{r}\right)=\left(T_{1}^{p}-x_{0}^{p}\right)\left(T_{1}^{p}-x_{1}^{p}\right) \cdots\left(T_{1}^{p}-x_{d-1}^{p}\right) .
$$

The algebra $H_{r, p, n}\left(x_{0}, \ldots, x_{d-1} ; q\right)$ is the subalgebra of $H_{r, 1, n}\left(u_{1}, \ldots, u_{r} ; q\right)$ generated by the elements

$$
a_{0}=T_{1}^{p}, \quad a_{1}=T_{1}^{-1} T_{2} T_{1}, \quad a_{i}=T_{i}, \quad \text { for } 2 \leq i \leq n .
$$

Then

(a) $H_{2,2, n}(1 ; q)$ is the Iwahori-Hecke algebra of type $D_{n}$,

(b) If $\eta$ is a primitive $d$ th root of unity then $H_{r, p, n}\left(1, \eta, \cdots, \eta^{d-1} ; 1\right)$ is the group algebra $\mathbb{C} G(r, p, n)$.

(1.3) Affine braid groups of type A. There are three common ways of depicting affine braids $[\mathrm{Cr}],[\mathrm{GL}],[\mathrm{Jo}]$ :

(a) As braids in a (slightly thickened) cylinder,

(b) As braids in a (slightly thickened) annulus,

(c) As braids with a flagpole.

See Figure 1. The multiplication is by placing one cylinder on top of another, placing one annulus inside another, or placing one flagpole braid on top of another. These are equivalent formulations: an annulus can be made into a cylinder by turning up the edges, and a cylindrical braid can be made into a flagpole braid by putting a flagpole down the middle of the cylinder and pushing the pole over to the left so that the strings begin and end to its right.

The group formed by the affine braids with $n$ strands is the affine braid group of type A. The affine braid group $\mathcal{B}_{\infty, 1, n}$ is presented by generators $T_{2}, \ldots, T_{n}$ and $X^{\varepsilon_{1}}$ (see Figure 2) with relations

(B1) $T_{i} T_{j}=T_{j} T_{i}$

if $|i-j|>1$,

(B2) $T_{i} T_{i+1} T_{i}=T_{i+1} T_{i} T_{i+1}$,

for $2 \leq i \leq n-1$,

(BB) $X^{\varepsilon_{1}} T_{2} X^{\varepsilon_{1}} T_{2}=T_{2} X^{\varepsilon_{1}} T_{2} X^{\varepsilon_{1}}$,

(B1') $X^{\varepsilon_{1}} T_{i}=T_{i} X^{\varepsilon_{1}}$

for $3 \leq i \leq n$.

Inductively define $X^{\varepsilon_{i}} \in \mathcal{B}_{\infty, 1, n}$ by

$$
X^{\varepsilon_{i}}=T_{i} X^{\varepsilon_{i-1}} T_{i}, \quad 2 \leq i \leq n .
$$

By drawing pictures of the corresponding affine braids one can check that the $X^{\varepsilon_{i}}$ all commute with each other. View the symbols $\varepsilon_{i}$ as a basis of $\mathbb{R}^{n}$ so that

$$
\mathbb{R}^{n}=\sum_{i=1}^{n} \mathbb{R} \varepsilon_{i}, \quad \text { and let } \quad L=\sum_{i=1}^{n} \mathbb{Z} \varepsilon_{i} .
$$

The affine braid group $\mathcal{B}_{\infty, 1, n}$ contains a large abelian subgroup

$$
X=\left\{X^{\lambda} \mid \lambda \in L\right\}
$$


where $X^{\lambda}=\left(X^{\varepsilon_{1}}\right)^{\lambda_{1}} \cdots\left(X^{\varepsilon_{n}}\right)^{\lambda_{n}}$ for $\lambda=\lambda_{1} \varepsilon_{1}+\cdots+\lambda_{n} \varepsilon_{n} \in L$.

The pole winding number of an affine braid $b \in \mathcal{B}_{\infty, 1, n}$ is $\kappa(b)$ where $\kappa: \mathcal{B}_{\infty, 1, n} \rightarrow \mathbb{Z}$ is the group homomorphism defined by $\kappa\left(X^{\varepsilon_{1}}\right)=1$ and $\kappa\left(T_{i}\right)=0, \quad 2 \leq i \leq n$. The affine braid group $\mathcal{B}_{\infty, p, n}$ is the subgroup of $\mathcal{B}_{\infty, 1, n}$ of affine braids with pole winding number equal to $0(\bmod p)$,

$$
\mathcal{B}_{\infty, p, n}=\left\{b \in \mathcal{B}_{\infty, 1, n} \mid \kappa(b)=0(\bmod p)\right\}
$$

Define

$$
Q=\sum_{i=2}^{n} \mathbb{Z}\left(\varepsilon_{i}-\varepsilon_{i-1}\right) \quad \text { and } \quad L_{p}=Q+\sum_{i=1}^{n} p \mathbb{Z} \varepsilon_{i},
$$

for each nonnegative integer $p$. The lattice $L_{p}$ is a lattice of index $p$ in $L$. Then

$$
\mathcal{B}_{\infty, p, n}=\left\langle X^{\lambda}, T_{i} \mid \lambda \in L_{p}, 2 \leq i \leq n\right\rangle
$$

and the group $X^{L_{p}}=\left\langle X^{\lambda} \mid \lambda \in L_{p}\right\rangle$ is an abelian subgroup of $\mathcal{B}_{\infty, p, n}$.

(1.9) Affine Hecke algebras of type A. The affine Hecke algebra $H_{\infty, 1, n}$ (resp. $H_{\infty, p, n}$ ) is the quotient of the group algebra $\mathbb{C B}_{\infty, 1, n}\left(\right.$ resp. $\left.\mathbb{C B}_{\infty, p, n}\right)$ by the relations

$$
T_{i}^{2}=\left(q-q^{-1}\right) T_{i}+1, \quad 2 \leq i \leq n .
$$

Let $L$ and $X^{\lambda}$ be as in (1.5) and (1.6). The subalgebra

$$
\mathbb{C}[X]=\operatorname{span}\left\{X^{\lambda} \mid \lambda \in L\right\} \quad\left(\text { resp. } \mathbb{C}\left[X^{L_{p}}\right]=\operatorname{span}\left\{X^{\lambda} \mid \lambda \in L_{p}\right\}\right)
$$

is a commutative subalgebra of $H_{\infty, 1, n}\left(\right.$ resp. $\left.H_{\infty, p, n}\right)$. The symmetric group $S_{n}$ acts on the lattice $L$ by permuting the $\varepsilon_{i}$ and the lattices $L_{p}$ are $S_{n}$-invariant sublattices of $L$. Let $s_{i}=(i, i-1) \in S_{n}$ and $\alpha_{i}=\varepsilon_{i}-\varepsilon_{i-1}$. For $2 \leq i \leq n$ and $\lambda \in L\left(\right.$ resp. $\left.\lambda \in L_{p}\right)$,

$$
X^{\lambda} T_{i}=X^{s_{i} \lambda} T_{i}+\left(q-q^{-1}\right) \frac{X^{\lambda}-X^{s_{i} \lambda}}{1-X^{-\alpha_{i}}}
$$

as elements of $H_{\infty, 1, n}$ (resp. $\left.H_{\infty, p, n}\right)$.

For each element $w \in S_{n}$ define $T_{w}=T_{i_{1}} \cdots T_{i_{p}}$ if $w=s_{i_{1}} \cdots s_{i_{p}}$ is an expression of $w$ as a product of simple reflections $s_{i}$ such that $p$ is minimal. The element $T_{w}$ does not depend on the choice of the reduced expression of $w$ [Bou, Ch. IV $\S 2$ Ex. 23]. The sets

$$
\left\{X^{\lambda} T_{w} \mid \lambda \in L, w \in S_{n}\right\} \quad \text { and } \quad\left\{X^{\lambda} T_{w} \mid \lambda \in L_{p}, w \in S_{n}\right\}
$$

are bases of $H_{\infty, 1, n}$ and $H_{\infty, p, n}$, respectively [Lu]. The center of $H_{\infty, 1, n}$ is

$$
Z\left(H_{\infty, 1, n}\right)=\mathbb{C}\left[X^{\varepsilon_{1}}, \ldots, X^{\varepsilon_{n}}\right]^{S_{n}}=\mathbb{C}[X]^{S_{n}},
$$

and $\mathbb{C}\left[X^{L_{p}}\right]^{S_{n}}$ is the center of $H_{\infty, p, n}$ (see Theorem 4.12 below).

\section{RePRESENTATION THEORY TRANSFER}


In this section we provide the mechanism for obtaining the representation theory of $H_{\infty, p, n}$ from $H_{\infty, 1, n}$ and for obtaining the representation theory of cyclotomic Hecke algebras from affine Hecke algebras. In order to obtain the representation theory of $H_{\infty, p, n}$ from $H_{\infty, 1, n}$ we identify $H_{\infty, p, n}$ as the set of fixed points of a certain group $G$ acting on $H_{\infty, 1, n}$ by automorphisms. Once this is done, the extended version of Clifford theory given in the Appendix allows one to construct the representations of $H_{\infty, p, n}$ from those of $H_{\infty, 1, n}$. The same technique can be applied to obtain the representations of the braid groups $\mathcal{B}_{\infty, p, n}$ from those of $\mathcal{B}_{\infty, 1, n}$, of the complex reflection groups $G(r, p, n)$ from those of $G(r, 1, n)$, and of the Weyl groups $W D_{n}$ from those of the Weyl groups $W B_{n}$.

(2.1) Obtaining $H_{\infty, p, n}$-modules from $H_{\infty, 1, n}$-modules. The following result is what is needed to apply the Clifford theory developed in the Appendix to derive the representation theory of the algebras $H_{\infty, p, n}$ from that of $H_{\infty, 1, n}$.

Theorem 2.2. Let $\xi$ be a primitive $p$ th root of unity. The algebra automorphism $g: H_{\infty, 1, n} \rightarrow$ $H_{\infty, 1, n}$ defined by

$$
g\left(X^{\varepsilon_{1}}\right)=\xi X^{\varepsilon_{1}}, \quad \text { and } \quad g\left(T_{i}\right)=T_{i}, \quad 2 \leq i \leq n,
$$

gives rise to an action of the group $\mathbb{Z} / p \mathbb{Z}=\left\{1, g, \ldots, g^{p-1}\right\}$ on $H_{\infty, 1, n}$ by algebra automorphisms and

$$
H_{\infty, p, n}=\left(H_{\infty, 1, n}\right)^{\mathbb{Z} / p \mathbb{Z}}
$$

is the set of fixed points of the $\mathbb{Z} / p \mathbb{Z}$-action.

Proof. Immediate from the definitions of $H_{\infty, 1, n}, H_{\infty, p, n}$ and (1.6).

The action of $\mathbb{Z} / p \mathbb{Z}$ on $H_{\infty, 1, n}$ which is given in Theorem 2.2 induces an action of $\mathbb{Z} / p \mathbb{Z}$ on the simple $H_{\infty, 1, n}$-modules, see (A.1) in the Appendix. The stabilizer $K$ of the action of $\mathbb{Z} / p \mathbb{Z}$ on a simple $H_{\infty, 1, n}$-module $M$ is the inertia group $K$ of $M$. The action of $K$ commutes with the action of $H_{\infty, p, n}$ on $M$ and we have a decomposition

$$
M \cong \bigoplus_{j=0}^{|K|-1} M^{(j)} \otimes K^{(j)},
$$

where $K^{(j)}, 1 \leq j \leq|K|-1$, are the simple $K$-modules and $M^{(j)}$ are $H_{\infty, p, n \text {-modules. Theo- }}$ rem A.13 of the Appendix shows that the $M^{(j)}$ are simple $H_{\infty, p, n}$-modules and that all simple $H_{\infty, p, n}$-modules are constructed in this way. In Theorem 3.15 we show that this method gives a combinatorial construction of the module $M^{(j)}$ in any case when the Young tableau theory is available. This is a generalization of the method used in [Ar2] and [HR].

(2.4) The surjective algebra homomorphisms $\Phi$ and $\Phi_{p}$. The homomorphisms $\Phi$ and $\Phi_{p}$ described below are the primary tools for transferring results from the affine Hecke algebras to cyclotomic Hecke algebras. Many results are easier to prove for affine Hecke algebras because of the large commutative subalgebra $\mathbb{C}[X]$ which is available in the affine Hecke algebra. The homomorphism $\Phi$ has also been used by Cherednik [Ch2], Ariki [Ar3] and many others.

Proposition 2.5. Let $H_{\infty, 1, n}$ be the affine Hecke algebra of type $A$ defined in (1.9) and let $H_{r, 1, n}\left(u_{1}, \ldots, u_{r} ; q\right)$ denote the cyclotomic Hecke algebra of (1.1). 
(a) Fix $u_{1}, \ldots, u_{r} \in \mathbb{C}, q \in \mathbb{C}^{*}$. There is a surjective algebra homomorphism given by

$$
\begin{aligned}
& \Phi: \quad H_{\infty, 1, n} \longrightarrow H_{r, 1, n}\left(u_{1}, \ldots, u_{r} ; q\right) \\
& T_{i} \longmapsto \quad T_{i}, \quad 2 \leq i \leq n, \\
& X^{\varepsilon_{1}} \longmapsto \quad T_{1} \text {. }
\end{aligned}
$$

(b) Restricting the homomorphism $\Phi$ to $H_{\infty, p, n}$ yields a surjective homomorphism defined by

$$
\begin{aligned}
\Phi_{p}: H_{\infty, p, n} & \longmapsto & H_{r, p, n}\left(x_{0}, \ldots, x_{d-1} ; q\right) & \\
T_{i} & \longmapsto & a_{i}, & 2 \leq i \leq n, \\
X^{p \varepsilon_{1}} & \longmapsto & a_{0}, & \\
X^{\varepsilon_{2}-\varepsilon_{1}} & \longmapsto & a_{1} a_{2} . &
\end{aligned}
$$

Proof. The result follows directly from the definitions of the affine Hecke algebras $H_{\infty, 1, n}$ and $H_{\infty, p, n}$ (see (1.9)) and the cyclotomic Hecke algebras $H_{r, 1, n}$ and $H_{r, p, n}$ (see (1.1) and (1.2)).

Let $L_{p}$ be the lattice defined in (1.8) and define $C_{r}=\left\{\lambda=\sum_{i=1}^{n} \lambda_{i} \varepsilon_{i} \mid 0 \leq \lambda_{i}<r\right\}$. Ariki and Koike $[\mathrm{AK}]$ and Ariki [Ar2] have shown that the sets

$$
\left\{\Phi\left(X^{\lambda} T_{w}\right) \mid \lambda \in C_{r}, w \in S_{n}\right\} \quad \text { and } \quad\left\{\Phi_{p}\left(X^{\lambda} T_{w}\right) \mid \lambda \in C_{r} \cap L_{p}, w \in S_{n}\right\}
$$

are bases of $H_{r, 1, n}$ and $H_{r, p, n}$, respectively.

(2.7) Relating $H_{\infty, 1, n}$-modules and $H_{r, 1, n}$-modules. The representation theory of the affine Hecke algebra $H_{\infty, 1, n}$ is equivalent to the representation theory of the cyclotomic Hecke algebras $H_{r, 1, n}$ (considering all possible $u_{1}, \ldots, u_{r} \in \mathbb{C}$ ). The elementary constructions in the following theorem allow us to make $H_{\infty, 1, n}$-modules into $H_{r, 1, n}$-modules and vice versa.

Theorem 2.8. Let $H_{\infty, 1, n}$ be the affine Hecke algebra of type $A$ defined in (1.9) and let $H_{r, 1, n}\left(u_{1}, \ldots, u_{r} ; q\right)$ be the cyclotomic Hecke algebra of (1.1).

(a) Fix $u_{1}, \ldots, u_{r} \in \mathbb{C}, q \in \mathbb{C}^{*}$, and let $\Phi$ be the surjective homomorphism of Proposition 2.5. If $M$ is a simple $H_{r, 1, n}\left(u_{1}, \ldots, u_{r} ; q\right)$-module then defining

$$
h m=\Phi(h) m, \quad \text { for all } h \in H_{\infty, 1, n} \text { and all } m \in M,
$$

makes $M$ into a simple $H_{\infty, 1, n}$-module.

(b) Let $M$ be a simple $H_{\infty, 1, n}$-module and let $\rho: H_{\infty, 1, n} \rightarrow \operatorname{End}(M)$ be the corresponding representation. Let $u_{1}, \ldots, u_{r} \in \mathbb{C}$ be such that the minimal polynomial $p(t)$ of the matrix $\rho\left(X^{\varepsilon_{1}}\right)$ divides the polynomial $\left(t-u_{1}\right) \cdots\left(t-u_{r}\right)$. Define an action of $H_{r, 1, n}\left(u_{1}, \ldots, u_{r} ; q\right)$ on $M$ by

$$
T_{1} m=X^{\varepsilon_{1}} m \quad \text { and } \quad T_{i} m=T_{i} m, \quad 2 \leq i \leq n,
$$

for all $m \in M$. Then $M$ is a simple $H_{r, 1, n}\left(u_{1}, \ldots, u_{r} ; q\right)$-module.

Proof. The Theorem follows directly from the definitions of $H_{\infty, 1, n}$ and $H_{r, 1, n}\left(u_{1}, \ldots, u_{r} ; q\right)$, and the construction of the surjective homomorphism $\Phi$.

Remark 2.9. The same translations work for arbitrary finite dimensional modules; in particular, they work for indecomposable modules and preserve composition series.

\section{Standard Young tableaux, Representations and Jucys-Murphy elements}


In this section we review the generalization of standard Young tableaux in [Ra5] which is used to construct representations of the affine Hecke algebras $H_{\infty, 1, n}$. Then we show how this theory can be transported to provide combinatorial constructions of simple modules for the cyclotomic Hecke algebras $H_{r, 1, n}\left(u_{1}, \ldots, u_{r} ; q\right)$ and $H_{r, p, n}\left(x_{0}, \ldots, x_{d-1} ; q\right)$. This approach shows that Jucy-Murphy type elements in the cyclotomic Hecke algebras arise naturally as images of the elements $X^{\varepsilon_{i}}$ in the affine Hecke algebra. The standard Jucys-Murphy type theorems then follow almost immediately from standard affine Hecke algebra facts.

(3.1) Skew shapes and standard tableaux. A partition $\lambda$ is a collection of $n$ boxes in a corner. We shall conform to the conventions in [Mac] and assume that gravity goes up and to the left.

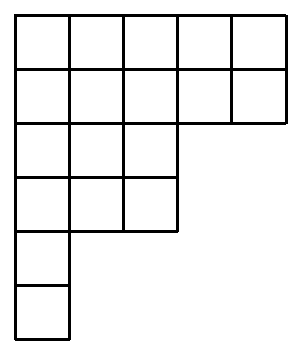

Any partition $\lambda$ can be identified with the sequence $\lambda=\left(\lambda_{1} \geq \lambda_{2} \geq \ldots\right)$ where $\lambda_{i}$ is the number of boxes in row $i$ of $\lambda$. The rows and columns are numbered in the same way as for matrices. In the example above we have $\lambda=(553311)$. If $\lambda$ and $\mu$ are partitions such that $\mu_{i} \leq \lambda_{i}$ for all $i$ we write $\mu \subseteq \lambda$. The skew shape $\lambda / \mu$ consists of all boxes of $\lambda$ which are not in $\mu$. Any skew shape is a union of connected components. Number the boxes of each skew shape $\lambda / \mu$ along major diagonals from southwest to northeast and

write $\operatorname{box}_{i}$ to indicate the box numbered $i$.

Let $\lambda / \mu$ be a skew shape with $n$ boxes. A standard tableau of shape $\lambda / \mu$ is a filling of the boxes in the skew shape $\lambda / \mu$ with the numbers $1, \ldots, n$ such that the numbers increase from left to right in each row and from top to bottom down each column.

(3.2) Placed skew shapes. Let $\mathbb{R}+i\left[0,2 \pi / \ln \left(q^{2}\right)\right)=\left\{a+b i \mid a \in \mathbb{R}, 0 \leq b \leq 2 \pi / \ln \left(q^{2}\right)\right\} \subseteq \mathbb{C}$. If $q$ is a positive real number then the function

$$
\begin{array}{cll}
\mathbb{R}+i\left[0,2 \pi / \ln \left(q^{2}\right)\right) & \longrightarrow \mathbb{C}^{*} \\
x & \longmapsto & q^{2 x}=e^{\ln \left(q^{2}\right) x}
\end{array}
$$

is a bijection. The elements of $[0,1)+i\left[0,2 \pi / \ln \left(q^{2}\right)\right)$ index the $\mathbb{Z}$-cosets in $\mathbb{R}+i\left[0,2 \pi / \ln \left(q^{2}\right)\right)$.

A placed skew shape is a pair $(c, \lambda / \mu)$ consisting of a skew shape $\lambda / \mu$ and a content function

$$
c:\{\text { boxes of } \lambda / \mu\} \longrightarrow \mathbb{R}+i\left[0,2 \pi / \ln \left(q^{2}\right)\right) \quad \text { such that }
$$

$c\left(\operatorname{box}_{j}\right)-c\left(\operatorname{box}_{i}\right) \geq 0, \quad$ if $i<j$ and $c\left(\operatorname{box}_{j}\right)-c\left(\operatorname{box}_{i}\right) \in \mathbb{Z}$,

$c\left(\operatorname{box}_{j}\right)=c\left(\operatorname{box}_{i}\right)+1, \quad$ if and only if $\operatorname{box}_{i}$ and $\operatorname{box}_{j}$ are on adjacent diagonals, and

$c\left(\operatorname{box}_{i}\right)=c\left(\operatorname{box}_{j}\right), \quad$ if and only if $\operatorname{box}_{i}$ and $\operatorname{box}_{j}$ are on the same diagonal.

This is a generalization of the usual notion of the content of a box in a partition (see [Mac] I $\S 1$ Ex. 3).

Suppose that $(c, \lambda / \mu)$ is a placed skew shape such that $c$ takes values in $\mathbb{Z}$. One can visualize $(c, \lambda / \mu)$ by placing $\lambda / \mu$ on a piece of infinite graph paper where the diagonals of the graph paper 
are indexed consecutively (with elements of $\mathbb{Z}$ ) from southeast to northwest. The content of a box $b$ is the index $c(b)$ of the diagonal that $b$ is on. In the general case, when $c$ takes values in $\mathbb{R}+i\left[0,2 \pi / \ln \left(q^{2}\right)\right)$, one imagines a book where each page is a sheet of infinite graph paper with the diagonals indexed consecutively (with elements of $\mathbb{Z}$ ) from southeast to northwest. The pages are numbered by values $\beta \in[0,1)+i\left[0,2 \pi / \ln \left(q^{2}\right)\right)$ and there is a skew shape $\lambda^{(\beta)} / \mu^{(\beta)}$ placed on page $\beta$. The skew shape $\lambda / \mu$ is a union of the disjoint skew shapes on each page,

$$
\lambda / \mu=\bigsqcup_{\beta}\left(\lambda^{(\beta)} / \mu^{(\beta)}\right), \quad \beta \in[0,1)+i\left[0,2 \pi / \ln \left(q^{2}\right)\right),
$$

and the content function is given by

$$
c(b)=(\text { page number of the page containing } b)+(\text { index of the diagonal containing } b) \text {. }
$$

for a box $b \in \lambda / \mu$.

(3.6) Example. The following diagrams illustrate standard tableaux and the numbering of boxes in a skew shape $\lambda / \mu$.

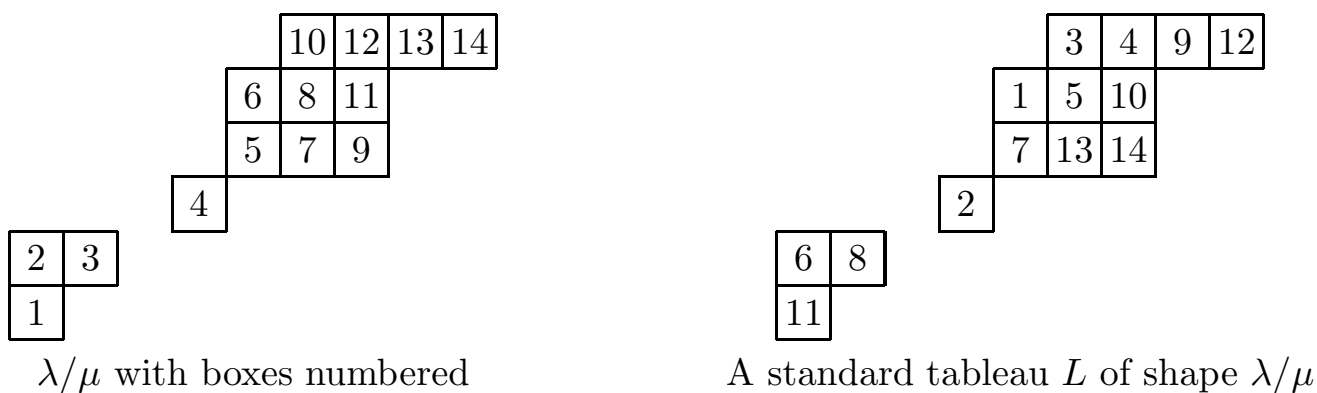

The following picture shows the contents of the boxes in the placed skew shape $(c, \lambda / \mu)$ such that the sequence $\left(c\left(\operatorname{box}_{1}\right), \ldots, c\left(\operatorname{box}_{n}\right)\right)$ is $(-7,-6,-5,-2,0,1,1,2,2,3,3,4,5,6)$.

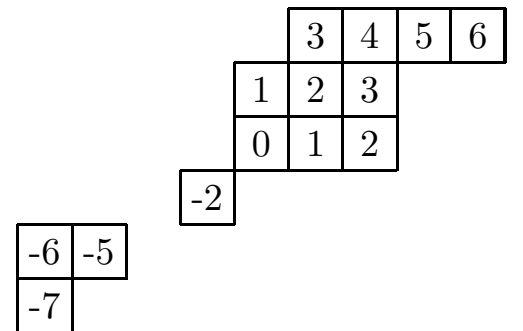

Contents of the boxes of $(c, \lambda / \mu)$

The following picture shows the contents of the boxes in the placed skew shape $(c, \lambda / \mu)$ such that $\left(c\left(\operatorname{box}_{1}\right), \ldots, c\left(\operatorname{box}_{n}\right)\right)=(-7,-6,-5,-3 / 2,1 / 2,3 / 2,3 / 2,5 / 2,5 / 2,7 / 2,7 / 2,9 / 2,11 / 2,13 / 2)$.

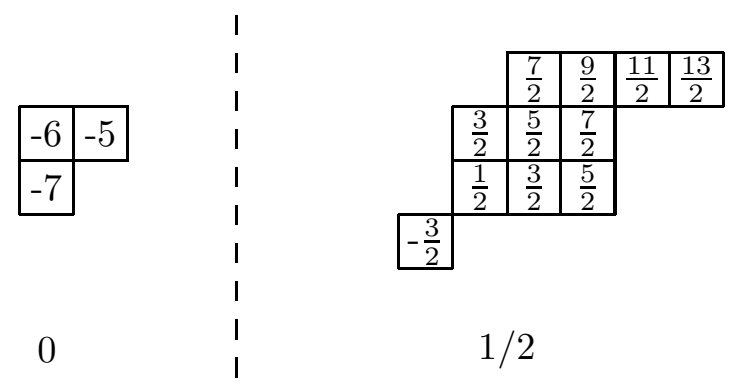


This "book" has two pages, with page numbers 0 and $1 / 2$.

(3.7) Calibrated $H_{\infty, 1, n}$-modules. A finite dimensional $\tilde{H}_{\infty, 1, n}$-module $M$ is calibrated if it has a basis $\left\{v_{t}\right\}$ such that for each $\lambda \in L$ and each $v_{t}$ in the basis

$$
X^{\lambda} v_{t}=t\left(X^{\lambda}\right) v_{t}, \quad \text { for some } t\left(X^{\lambda}\right) \in \mathbb{C} .
$$

This is the class of representations of the affine Hecke algebra for which there is a good theory of Young tableaux [Ra2].

The following theorem classifies and constructs all irreducible calibrated representations of the affine Hecke algebra $H_{\infty, 1, n}$. The construction is a direct generalization of A. Young's classical "seminormal construction" of the irreducible representations of the symmetric group [Yg2]. Young's construction was generalized to Iwahori-Hecke algebras of type A by Hoefsmit [Hf] and Wenzl [Wz] independently, to Iwahori-Hecke algebras of types B and D by Hoefsmit [Hf] and to cyclotomic Hecke algebras by Ariki and Koike [AK]. In (3.7) and (3.11) below we show how all of these earlier generalizations of Young's construction can be obtained from Theorem 3.8. Some parts of Theorem 3.8 are originally due to I. Cherednik, and are stated in [Ch1, 3$]$.

Theorem 3.8. ([Ra, Theorem 4.1]) Let $(c, \lambda / \mu)$ be a placed skew shape with $n$ boxes. Define an action of $H_{\infty, 1, n}$ on the vector space

$$
\tilde{H}^{(c, \lambda / \mu)}=\mathbb{C}-\operatorname{span}\left\{v_{L} \mid L \text { is a standard tableau of shape } \lambda / \mu\right\}
$$

by the formulas

$$
\begin{aligned}
X^{\varepsilon_{i}} v_{L} & =q^{2 c(L(i))} v_{L}, \\
T_{i} v_{L} & =\left(T_{i}\right)_{L L} v_{L}+\left(q^{-1}+\left(T_{i}\right)_{L L}\right) v_{s_{i} L},
\end{aligned}
$$

where $s_{i} L$ is the same as $L$ except that the entries $i-1$ and $i$ are interchanged,

$$
\left(T_{i}\right)_{L L}=\frac{q-q^{-1}}{1-q^{2(c(L(i-1))-c(L(i)))}}, \quad v_{s_{i} L}=0 \text { if } s_{i} L \text { is not a standard tableau }
$$

and $L(i)$ denotes the box of $L$ containing the entry $i$.

(a) $\tilde{H}^{(c, \lambda / \mu)}$ is a calibrated irreducible $H_{\infty, 1, n}$-module.

(b) The modules $\tilde{H}^{(c, \lambda / \mu)}$ are non-isomorphic.

(c) Every irreducible calibrated $H_{\infty, 1, n}$-module is isomorphic to $\tilde{H}^{(c, \lambda / \mu)}$ for some placed skew shape $(c, \lambda / \mu)$.

Remark 3.9. All of the irreducible modules for the affine Hecke algebra have been classified and constructed by Kazhdan and Lusztig [KL]. The construction in [KL] is geometric and noncombinatorial. It is nontrivial to relate the construction of Theorem 3.8 and the classification in [KL]. This point is the subject of $[R R]$.

(3.10) Calibrated $H_{\infty, p, n}$-modules. A finite dimensional $H_{\infty, p, n}$ module $M$ is calibrated if it has a basis $\left\{v_{t}\right\}$ such that for each $\lambda \in L_{p}$ and each $v_{t}$ in the basis

$$
X^{\lambda} v_{t}=t\left(X^{\lambda}\right) v_{t}, \quad \text { for some } t\left(X^{\lambda}\right) \in \mathbb{C} .
$$


Let us show how Theorem 3.8, Theorem 2.2 and Theorem A.13 provide explicit constructions of simple calibrated $H_{\infty, p, n}$-modules. The resulting construction is a generalization of the construction of $H_{r, p, n}\left(x_{0}, \ldots, x_{d-1} ; q\right)$-modules given by Ariki [Ar2] (as amplified and applied in [HR]). Comparing the following machinations with those in $[\mathrm{HR}, \S 3]$ (where more pictures are given) will be helpful.

The $(\mathbb{Z} / p \mathbb{Z})$-action on $H_{\infty, 1, n}$ induces an action of $\mathbb{Z} / p \mathbb{Z}$ on the simple $H_{\infty, 1, n}$-modules, as in (A.1) of the Appendix, and this action takes calibrated modules to calibrated modules since the $(\mathbb{Z} / p \mathbb{Z})$-action on $H_{\infty, 1, n}$ preserves the subalgebra $\mathbb{C}[X]$. The $\mathbb{Z} / p \mathbb{Z}$ action on simple calibrated modules can be described combinatorially as follows.

If $(c, \lambda / \mu)$ is a placed skew shape with $n$ boxes and $g \in \mathbb{Z} / p \mathbb{Z}$ define

$$
g(c, \lambda / \mu)=(c-i \alpha / p, \lambda / \mu), \quad \text { where } \alpha=2 \pi / \ln \left(q^{2}\right),
$$

and $c-i \alpha / p$ denotes the content function defined by $(c-i \alpha / p)(b)=c(b)-i \alpha / p$, for all boxes $b \in \lambda / \mu$. To make this definition we are identifying the set $[0, \alpha)$ with $\mathbb{R} / \alpha \mathbb{Z}$. One can imagine the placed skew shape as a book with pages numbered by values $\beta \in[0,1)+i(\mathbb{R} / \alpha \mathbb{Z})$ and a skew shape $\lambda^{(\beta)} / \mu^{(\beta)}$ on each page. The action of

$$
g \text { cyclically permutes the pages numbered } \beta+i(k / p) \alpha, \quad 0 \leq k<p \text {. }
$$

If $L$ is a standard tableau of shape $(c, \lambda / \mu)$ let $g L$ denote the same filling of $\lambda / \mu$ as $L$ but viewed as a standard tableaux of shape $g(c, \lambda / \mu)$.

Let ${ }^{g} \tilde{H}^{(c, \lambda / \mu)}$ be the $H_{\infty, 1, n}$-module $\tilde{H}^{(c, \lambda / \mu)}$ except twisted by the automorphism $g$, see (A.1) in the Appendix. It follows from the formulas in Theorem 3.8 that the map

$$
\begin{aligned}
\phi: \tilde{H}^{(c, \lambda / \mu)} & \longrightarrow \tilde{H}^{g(c, \lambda / \mu)} \\
v_{L} & \longmapsto v_{g L}
\end{aligned}
$$

is an $H_{\infty, 1, n}$-module isomorphism. Indeed, since $g^{-1}\left(T_{j}\right)=T_{j}$ and $\left(T_{j}\right)_{g L, g L}=\left(T_{j}\right)_{L L}$,

$$
\begin{aligned}
\phi\left(T_{j} \circ v_{L}\right) & =T_{j} \phi\left(v_{L}\right), \quad \text { and } \\
\phi\left(X^{\varepsilon_{j}} \circ v_{L}\right) & =\phi\left(g^{-1}\left(X^{\varepsilon_{j}}\right) v_{L}\right)=\phi\left(\xi^{-1} X^{\varepsilon_{j}} v_{L}\right)=q^{2(c(L(j))-i \alpha / p)} v_{g L}=X^{\varepsilon_{j}} v_{g L}=X^{\varepsilon_{j}} \phi\left(v_{L}\right),
\end{aligned}
$$

where $\circ$ denotes the $H_{\infty, 1, n}$-action on ${ }^{g} \tilde{H}^{(c, \lambda / \mu)}$ as in (A.1). Identify ${ }^{g} \tilde{H}^{(c, \lambda / \mu)}$ with $\tilde{H}^{g(c, \lambda / \mu)}$ via the isomorphism in (3.12).

Let $(c, \lambda / \mu)$ be a placed skew shape with $n$ boxes and let $K_{(c, \lambda / \mu)}$ be the stabilizer of $(c, \lambda / \mu)$ under the action of $\mathbb{Z} / p \mathbb{Z}$. The cyclic group $K_{(c, \lambda / \mu)}$ is a realization of the inertia group of $\tilde{H}^{(c, \lambda / \mu)}$ and

$$
K_{(c, \lambda / \mu)}=\left\{\left(g^{\kappa}\right)^{\ell}: \tilde{H}^{(c, \lambda / \mu)} \rightarrow \tilde{H}^{(c, \lambda / \mu)}|0 \leq \ell \leq| K_{c, \lambda / \mu)} \mid-1\right\},
$$

where $\kappa$ is the smallest integer between 1 and $p$ such that $g^{\kappa}(c, \lambda / \mu)=(c, \lambda / \mu)$ and $\left|K_{(c, \lambda / \mu)}\right|$ is the order of $K_{(c, \lambda / \mu)}$. The elements of $K_{(c, \lambda / \mu)}$ are all $H_{\infty, p, n}$-module isomorphisms. Since $K_{(c, \lambda / \mu)}$ is a cyclic group the irreducible $K_{(c, \lambda / \mu)}$-modules are all one-dimensional and the characters of these modules are given explicitly by

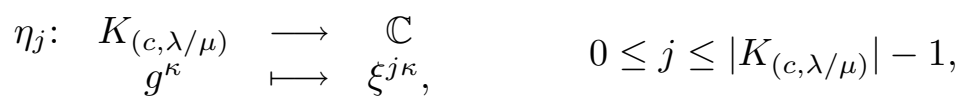

since $\xi^{\kappa}$ is a primitive $\left|K_{(c, \lambda / \mu)}\right|$-th root of unity. The element

$$
p_{j}=\sum_{\ell=0}^{\left|K_{(c, \lambda / \mu)}\right|-1} \xi^{-j \ell \kappa} g^{\ell \kappa} .
$$


is the minimal idempotent of the group algebra $\mathbb{C} K_{(c, \lambda / \mu)}$ corresponding to the irreducible character $\eta_{j}$. It follows (from a standard double centralizer result, [Bou2]) that, as an $\left(H_{\infty, p, n}, K_{(c, \lambda / \mu)}\right)$ bimodule,

$$
\tilde{H}^{(c, \lambda / \mu)} \cong \bigoplus_{j=0}^{\mid K_{(c, \lambda / \mu) \mid-1}} \tilde{H}^{(c, \lambda / \mu, j)} \otimes K^{(j)}, \quad \text { where } \quad \tilde{H}^{(c, \lambda / \mu, j)}=p_{j} \tilde{H}^{(c, \lambda / \mu)}
$$

and $K^{(j)}$ is the irreducible $K_{(c, \lambda / \mu)}$-module with character $\eta_{j}$. The following theorem now follows from Theorem A.13 of the Appendix.

Theorem 3.15. Let $(c, \lambda / \mu)$ be a placed skew shape with $n$ boxes and let $\tilde{H}^{(c, \lambda / \mu)}$ be the simple calibrated $H_{\infty, 1, n}$-module constructed in Theorem 3.8. Let $K_{(c, \lambda / \mu)}$ be the inertia group of $\tilde{H}^{(c, \lambda / \mu)}$ corresponding to the action of $\mathbb{Z} / p \mathbb{Z}$ on $H_{\infty, 1, n}$ defined by (A.1). If $p_{j}$ is the minimal idempotent of $K_{(c, \lambda / \mu)}$ given by (3.13) then

$$
\tilde{H}^{(c, \lambda / \mu, j)}=p_{j} \tilde{H}^{(c, \lambda / \mu)}
$$

is a simple calibrated $H_{\infty, p, n}$-module.

Theorem 3.15 provides a generalization of the construction of the $H_{r, p, n}\left(x_{0}, \ldots, x_{d-1} ; q\right)$ modules which was given by Ariki [Ar2] and extended and applied in [HR].

(3.16) Simple $H_{r, 1, n}\left(u_{1}, \ldots, u_{r} ; q\right)$-modules. Many (usually all) of the simple $H_{r, 1, n}\left(u_{1}, \ldots, u_{r} ; q\right)$ modules can be constructed with Theorems 2.8 and 3.8 .

If $\lambda / \mu$ is a skew shape define

$$
N W(\lambda / \mu)=\{\text { northwest corner boxes of } \lambda / \mu\},
$$

so that $N W(\lambda / \mu)$ is the set of boxes $b \in \lambda / \mu$ such that there is no box of $\lambda / \mu$ immediately above or immediately to the left of $b$.

Theorem 3.17. Fix $u_{1}, \ldots, u_{r} \in \mathbb{C}^{*}$ and let $(c, \lambda / \mu)$ be a placed skew shape with $n$ boxes. If

$$
\left\{q^{2 c(b)} \mid b \in N W(\lambda / \mu)\right\} \subseteq\left\{u_{1}, \ldots, u_{r}\right\}
$$

then the $H_{\infty, 1, n}$-module $\tilde{H}^{(c, \lambda / \mu)}$ is a simple $H_{r, 1, n}\left(u_{1}, \ldots, u_{r} ; q\right)$-module (via Theorem 2.8).

Proof. Let $(c, \lambda / \mu)$ be a placed skew shape with $n$ boxes and let $\tilde{H}^{(c, \lambda / \mu)}$ be the simple $H_{\infty, 1, n^{-}}$ module of Theorem 3.8. Let $\rho^{(c, \lambda / \mu)}: H_{\infty, 1, n} \rightarrow \operatorname{End}\left(\tilde{H}^{(c, \lambda / \mu)}\right)$ be the representation corresponding to $\tilde{H}^{(c, \lambda / \mu)}$. By the formulas in Theorem 3.8 the matrix $\rho\left(X^{\varepsilon_{1}}\right)$ is diagonal with eigenvalues $q^{2 c(L(1))}$, for $L \in \mathcal{F}^{(c, \lambda / \mu)}$, where $\mathcal{F}^{(c, \lambda / \mu)}$ is the set of standard tableaux of shape $\lambda / \mu$.

The boxes $L(1), L \in \mathcal{F}^{(c, \lambda / \mu)}$ are exactly the northwest corner boxes of $\lambda / \mu$ and so the minimal polynomial of $\rho\left(X^{\varepsilon_{1}}\right)$ is

$$
p(t)=\prod_{b \in N W(\lambda / \mu)}\left(t-q^{2 c(b)}\right) .
$$

Thus the condition $\left\{q^{2 c(b)} \mid b \in N W(\lambda / \mu)\right\} \subseteq\left\{u_{1}, \ldots, u_{r}\right\}$ is exactly what is needed for $\tilde{H}^{(c, \lambda / \mu)}$ to be an $H_{r, 1, n}\left(u_{1}, \ldots, u_{r} ; q\right)$-module via Theorem 2.8 . 
Theorem 3.18. If the cyclotomic Hecke algebra $H_{r, 1, n}\left(u_{1}, u_{2}, \ldots, u_{r} ; q\right)$ is semisimple, then its simple modules are the modules $\tilde{H}^{(c, \lambda)}$ constructed in Theorem 3.17 , where $\lambda=\left(\lambda^{(1)}, \ldots, \lambda^{(r)}\right)$ is an $r$-tuple of partitions with a total of $n$ boxes and $c$ is the content function determined by

$$
q^{2 c(b)}=u_{i}, \quad \text { if } b \text { is the northwest corner box of } \lambda^{(i)} .
$$

Proof. The equations $q^{2 c(b)}=u_{i}$, for $b \in N W\left(\lambda^{(i)}\right)$, determine the values $c(b)$ for all boxes $b \in \lambda$ and thus the pair $(c, \lambda)$ defines a placed skew shape. By a Theorem of Ariki [Ar1], $H_{r, 1, n}\left(u_{1}, \ldots, u_{r} ; q\right)$ is semisimple if and only if

$$
[n]_{q} ! \neq 0 \quad \text { and } \quad u_{i} u_{j}^{-1} \notin\left\{1, q^{2}, \ldots, q^{2 n}\right\}
$$

where $[n]_{q} !=[n]_{q}[n-1]_{q} \cdots[2]_{q}[1]_{q}$ and $[k]_{q}=\left(q^{k}-q^{-k}\right) /\left(q-q^{-1}\right)$. These conditions guarantee that $(c, \lambda)$ is a placed skew shape and that the $\tilde{H}_{\infty, 1, n}$-module $\tilde{H}^{(c, \lambda)}$ defined in Theorem 3.8 is well defined and irreducible. The reduction in Theorem 2.8 makes $\tilde{H}^{(c, \lambda)}$ into a simple $H_{r, 1, n}\left(u_{1}, \ldots, u_{r} ; q\right)$-module and a count of standard tableaux (using binomial coefficients and the classical identity for the symmetric group case) shows that

$$
\sum_{\lambda=\left(\lambda^{(1)}, \ldots, \lambda^{(r)}\right)} \operatorname{dim}\left(\tilde{H}^{(c, \lambda)}\right)^{2}=r^{n} n !=\operatorname{dim}\left(H_{r, 1, n}\right),
$$

where the sum is over all $r$-tuples $\lambda=\left(\lambda^{(1)}, \ldots, \lambda^{(r)}\right)$ with a total of $n$ boxes. Thus the $\tilde{H}^{(c, \lambda)}$ are a complete set of simple $H_{r, 1, n}\left(u_{1}, \ldots, u_{r} ; q\right)$-modules.

Theorem 3.18 demonstrates that the construction of simple modules for $H_{r, 1, n}\left(u_{1}, \ldots, u_{r} ; q\right)$ by Ariki and Koike [AK, Theorem 3.7], for $H_{2,1, n}\left(p,-p^{-1} ; q\right)$ by Hoefsmit [Hf], for $H_{1,1, n}(1 ; q)$ by Hoefsmit [Hf] and Wenzl [Wz] (independently), and for $H_{1,1, n}(1 ; 1)=\mathbb{C} S_{n}$ and $H_{2,1, n}(1,1 ; 1)=$ $\mathbb{C} W B_{n}$ by Young [Yg1-2], are all special cases of Theorem 3.8.

(3.19) Jucys-Murphy elements in cyclotomic Hecke algebras. The following result is well known, but we give a new proof which shows that the cyclotomic Hecke algebra analogues of the Jucys-Murphy elements which have appeared in the literature (see [BMM], [Ra1], [DJM] and the references there) come naturally from the affine Hecke algebra $H_{\infty, 1, n}$.

Corollary 3.20. Let $H_{r, 1, n}\left(u_{1}, \ldots, u_{r} ; q\right)$ and $H_{r, p, n}\left(x_{0}, \ldots, x_{d-1} ; q\right)$ be the cyclotomic Hecke algebras defined in (1.1) and (1.2).

(a) The elements

$$
M_{i}=T_{i} \cdots T_{2} T_{1} T_{2} \cdots T_{i}, \quad 1 \leq i \leq n,
$$

generate a commutative subalgebra of $H_{r, 1, n}\left(u_{1}, \ldots, u_{r} ; q\right)$.

(b) If $H_{r, 1, n}\left(u_{1}, \ldots, u_{r} ; q\right)$ is semisimple then every simple $H_{r, 1, n}\left(u_{1}, \ldots, u_{r} ; q\right)$-module has a basis of simultaneous eigenvectors of the elements $M_{i}$.

(c) The elements

$$
M_{1}^{p}=a_{0}, \quad \text { and } \quad M_{i} M_{1}^{-1}=a_{i} \cdots a_{3} a_{2} a_{1} a_{3} \cdots a_{i}, \quad 2 \leq i \leq n,
$$

generate a commutative subalgebra of $H_{r, p, n}\left(x_{0}, \ldots, x_{d-1} ; q\right)$.

(d) If $H_{r, p, n}\left(x_{0}, \ldots, x_{d-1} ; q\right)$ is semisimple then every simple $H_{r, p, n}\left(x_{0}, \ldots, x_{d-1} ; q\right)$-module has a basis of simultaneous eigenvectors of the elements $M_{1}^{p}$ and $M_{i} M_{1}^{-1}, 2 \leq i \leq n$. 
Proof. (a) The elements $X^{\varepsilon_{i}}, 1 \leq i \leq n$, generate the subalgebra $\mathbb{C}[X] \subseteq H_{\infty, 1, n}$. Inductive use of the relation (1.4) shows that $M_{i}=\Phi\left(X^{\varepsilon_{i}}\right)$, where $\Phi: H_{\infty, 1, n} \rightarrow H_{r, 1, n}\left(u_{1}, \ldots, u_{r} ; q\right)$ is the homomorphism in Proposition 2.5. Thus the subalgebra of $H_{r, 1, n}\left(u_{1}, \ldots, u_{r} ; q\right)$ generated by the element $M_{i}$ is the image of $\mathbb{C}[X]$ under the homomorphism $\Phi$.

(b) is an immediate consequence of Theorem 3.18, the construction described in Theorem 3.8, and the fact that $M_{i}=\Phi\left(X^{\varepsilon_{i}}\right)$.

(c) The elements $X^{p \varepsilon_{1}}$ and $X^{\varepsilon_{i}-\varepsilon_{1}}, 2 \leq i \leq n$, generate the subalgebra $\mathbb{C}\left[X^{L_{p}}\right] \subseteq H_{\infty, p, n}$ and the images of these elements under the homomorphism $\Phi_{p}: H_{\infty, p, n} \rightarrow H_{r, p, n}\left(x_{0}, \ldots, x_{d-1} ; q\right)$ are the elements $M_{1}^{p}$ and $M_{i} M_{1}^{-1}$.

The proof of part (d) uses the construction described in Theorem 3.15 and is analogous to the proof of part (b).

(3.21) The center of $H_{r, 1, n}\left(u_{1}, \ldots, u_{r} ; q\right)$. It is an immediate consequence of $(1.12)$ and the proof of Corollary 3.20 that

$$
\mathbb{C}\left[M_{1}, \ldots, M_{n}\right]^{S_{n}} \subseteq Z\left(H_{r, 1, n}\left(u_{1}, \ldots, u_{r} ; q\right)\right),
$$

where $M_{i}=T_{i} T_{i-1} \cdots T_{2} T_{1} T_{2} \cdots T_{i-1} T_{i}$. The following proposition shows that this inclusion is an equality.

Proposition 3.22. If the cyclotomic Hecke algebra $H_{r, 1, n}\left(u_{1}, \ldots, u_{r} ; q\right)$ is semisimple then its center

$$
Z\left(H_{r, 1, n}\left(u_{1}, \ldots, u_{r} ; q\right)\right)=\mathbb{C}\left[M_{1}, \ldots, M_{n}\right]^{S_{n}},
$$

where $M_{i}=T_{i} T_{i-1} \cdots T_{2} T_{1} T_{2} \cdots T_{i-1} T_{i}$ and $\mathbb{C}\left[M_{1}, \ldots, M_{n}\right]^{S_{n}}$ is the ring of symmetric polynomials in $M_{1}, \ldots, M_{n}$.

Proof. By $(1.12) Z\left(H_{\infty, 1, n}\right)=\mathbb{C}[X]^{S_{n}}$. Thus, since $M_{i}=\Phi\left(X^{\varepsilon_{i}}\right)$, where $\Phi: H_{\infty, 1, n} \rightarrow H_{r, 1, n}\left(u_{1}, \ldots, u_{r} ; q\right)$ is the surjective homomorphism of Proposition 2.5, it follows that

$$
\mathbb{C}\left[M_{1}, \ldots, M_{n}\right]^{S_{n}}=\Phi\left(\mathbb{C}[X]^{S_{n}}\right)=\Phi\left(Z\left(H_{\infty, 1, n}\right)\right) \subseteq Z\left(H_{r, 1, n}\left(u_{1}, \ldots, u_{r} ; q\right)\right) .
$$

For the reverse inclusion we need to show that the action of the elements $\mathbb{C}\left[M_{1}, \ldots, M_{n}\right]^{S_{n}}$ distinguishes the simple $H_{r, 1, n}\left(u_{1}, \ldots, u_{r} ; q\right)$-modules.

Let $\lambda=\left(\lambda^{(1)}, \ldots, \lambda^{(r)}\right)$ be an $r$-tuple of partitions with a total of $n$ boxes and let $\tilde{H}^{(c, \lambda)}$ be the corresponding simple $H_{r, 1, n}\left(u_{1}, \ldots, u_{r} ; q\right)$-module as constructed by Theorem 3.18 (and Theorem 3.8). If $L$ is a standard tableau of shape $\lambda$ then $M_{1}, \ldots, M_{n}$ act on $v_{L}$ by the multiset of values $\left(q^{2 c(L(1))}, \ldots, q^{2 c(L(n))}\right)$. The elementary symmetric functions $e_{i}\left(M_{1}, \ldots, M_{n}\right)$ act on $\tilde{H}^{(c, \lambda)}$ by the values $a_{i}=e_{i}\left(q^{2 c(L(1))}, \ldots, q^{2 c(L(n))}\right)$. Note that $a_{i}$ does not depend on the choice of the standard tableau $L$ (since $e_{i}\left(M_{1}, \ldots, M_{n}\right) \in Z\left(H_{r, 1, n}\right)$ ). We show that the simple module $\tilde{H}^{(c, \lambda)}$ is determined by the values $a_{1}, \ldots, a_{n}$. This shows that the simple modules are distinguished by the elements of $\mathbb{C}\left[M_{1}, \ldots, M_{n}\right]^{S_{n}}$.

Let us explain how the values $a_{1}, \ldots, a_{n}$ determine the placed skew shape $(c, \lambda)$. There is a unique (unordered) multiset of values $b_{1}, \ldots, b_{n}$ such that $e_{i}\left(b_{1}, \ldots, b_{n}\right)=a_{i}$ for all $1 \leq i \leq n$. The $b_{i}$ are determined by the equation

$$
\left(t-b_{1}\right) \cdots\left(t-b_{n}\right)=t^{n}-a_{1} t^{n-1}+a_{2} t^{n-2}-a_{3} t^{n-3}+\cdots \pm a_{n} .
$$

From the previous paragraph, the multiset $\left\{b_{1}, \ldots, b_{n}\right\}$ must be the same as the multiset 
$\left\{q^{2 c(L(1))}, \ldots, q^{2 c(L(n))}\right\}$. In this way the multiset $S=\{c(L(1)), \ldots, c(L(n))\}$ is determined by $a_{1}, \ldots, a_{n}$.

The multiset $S$ is a disjoint union $S=S_{1} \sqcup \cdots \sqcup S_{r}$ of multisets such that each $S_{i}$ is in a single $\mathbb{Z}$-coset of $\mathbb{R}+i\left[0,2 \pi / \ln \left(q^{2}\right)\right) i$ (see (3.4)). These $\mathbb{Z}$ cosets are determined by the values $u_{1}, \ldots, u_{r}$ and thus there is a one-to-one correspondence between the $u_{i}$ and the multisets $S_{i}$. Then $\left|\lambda^{(i)}\right|=\operatorname{Card}\left(S_{i}\right)$, the nonempty diagonals of $\lambda^{(i)}$ are determined by the values in $S_{i}$, and the lengths of the diagonals of $\lambda^{(i)}$ are the multiplicities of the values of the elements of $S_{i}$. This information completely determines $\lambda^{(i)}$ for each $i$. Thus $(c, \lambda)$ is determined by the values $a_{1}, \ldots, a_{n}$.

Remark 3.23. In the language of affine Hecke algebra representations (see $[\mathrm{Ra} 2]$ ) the proof of Proposition 3.22 shows that (when $H_{r, 1, n}\left(u_{1}, \ldots, u_{r} ; q\right)$ is semisimple) the simple $H_{r, 1, n}\left(u_{1}, \ldots, u_{r} ; q\right)$ modules $\tilde{H}^{(c, \lambda)}$ all have different central characters (as $H_{\infty, 1, n}$ modules).

Remark 3.24. The elements $M_{1}^{p}$ and $M_{i} M_{1}^{-1}$ from Corollary 3.20 cannot be used to obtain a direct analogue of Proposition 3.22 for $H_{r, p, n}\left(x_{0}, \ldots, x_{d-1} ; q\right)$. This is because all of the $H_{\infty, p, n^{-}}$ modules $V_{j}$ appearing in the decomposition (3.14) will have the same central character. However, when $n$ is odd, the natural analogue of Proposition 3.22 does hold for Iwahori-Hecke algebras of type $D_{n}, H D_{n}(q)=H_{2,2, n}(1 ; q)$. In that case, every simple $H_{2,1, n}$-module has trivial inertia group and the decomposition in (3.14) has only one summand.

\section{Affine Hecke algebras of general type}

Let $R$ be a reduced root system and let $R^{\vee}$ be the root system formed by the coroots $\alpha^{\vee}=$ $2 \alpha /\langle\alpha, \alpha\rangle$, for $\alpha \in R$. Let $W$ be the Weyl group of $R$ and fix a system of positive roots $R^{+}$in $R$. Let $\left\{\alpha_{1}, \ldots, \alpha_{n}\right\}$ be the corresponding simple roots and let $s_{1}, \ldots, s_{n}$ be the corresponding simple reflections in $W$. The fundamental weights are defined by the equations $\left\langle\omega_{i}, \alpha_{j}^{\vee}\right\rangle=\delta_{i j}$ and the lattices

$$
P=\sum_{i=1}^{n} \mathbb{Z} \omega_{i} \quad \text { and } \quad Q=\sum_{i=1}^{n} \mathbb{Z} \alpha_{i}
$$

are the weight lattice and the root lattice, respectively. The Dynkin diagrams and the corresponding extended Dynkin diagrams are given in Figure 3. If $\Gamma$ is a Dynkin diagram or extended Dynkin diagram define

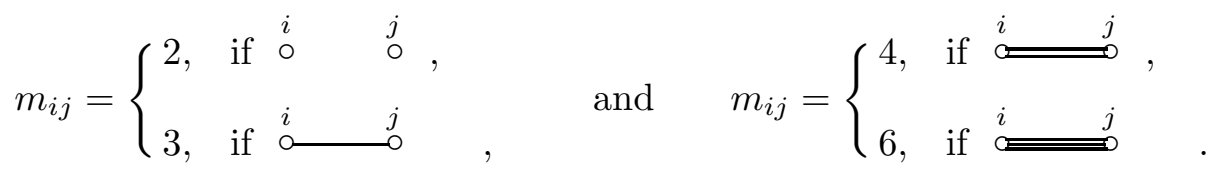

(4.1) Affine Weyl groups. The extended affine Weyl group is the group

$$
\tilde{W}=W \ltimes P=\left\{w t_{\lambda} \mid w \in W, \lambda \in P\right\} \text { nodes } \text { nodes } \quad \text { where } w t_{\lambda}=t_{w \lambda} w,
$$

for $w \in W$ and $\lambda \in P$ where $t_{\lambda}$ corresponds to translation by $\lambda \in P$. Define $s_{0} \in \tilde{W}$ by the equation

$$
s_{0} s_{\phi} \vee=t_{\phi}, \quad \text { where } \phi^{\vee} \text { is the highest root of } R^{\vee},
$$

see [Bou Ch. IV $\S 1$ no. 2.1]. The subgroup $W_{\text {aff }}=W \ltimes Q$ of $\tilde{W}$ is presented by generators $s_{0}, s_{1}, \ldots, s_{n}$ and relations

$$
s_{i}^{2}=1, \quad 0 \leq i \leq n, \quad \text { and } \quad \underbrace{s_{i} s_{j} \cdots}_{m_{i j}}=\underbrace{s_{j} s_{i} \cdots}_{m_{i j}}, \quad i \neq j,
$$


where the $m_{i j}$ are determined from the extended Dynkin diagram of the root system $R^{\vee}$. Define

$$
\Omega=\left\{g_{i} \mid \omega_{i} \text { is minuscule }\right\}, \quad \text { where } \quad g_{i} w_{0} w_{0, i}=t_{\omega_{i}},
$$

$w_{0}$ is the longest element of $W$ and $w_{0, i}$ is the longest element of the group $\left\langle s_{j} \mid 1 \leq j \leq n, j \neq i\right\rangle$, see [Bou Ch. IV $\S 2$ Prop. 6]. Then $\Omega \cong P / Q$ and each element $g \in \Omega$ corresponds to an automorphism of the extended Dynkin diagram of $R^{\vee}$, in the sense that

$$
\text { if } g \in \Omega \text { then } g s_{i} g^{-1}=s_{\sigma(i)},
$$

where $\sigma$ is the permutation of the nodes determined by the automorphism. Equation (4.4) means that $\tilde{W}=W_{\text {aff }} \rtimes \Omega$. The usual length function on the Coxeter group $W_{\text {aff }}$ is extended to the group $\tilde{W}$ by

$$
\ell(w g)=\ell(w), \quad \text { for } w \in W_{\text {aff }} \text { and } g \in \Omega .
$$

Let $L$ be a lattice such that $Q \subseteq L \subseteq P$. View $L / Q$ as a subgroup of $\Omega \cong P / Q$ and let

$$
\tilde{W}_{L}=W \ltimes L=W_{\text {aff }} \rtimes(L / Q) .
$$

Then $W_{\text {aff }}=\tilde{W}_{Q}, \tilde{W}=\tilde{W}_{P}$, and $\tilde{W}_{L}$ is a subgroup of $\tilde{W}$.

(4.5) Affine braid groups. Let $L$ be a lattice such that $Q \subseteq L \subseteq P$. The affine braid group $\tilde{\mathcal{B}}_{L}$ is the group given by generators $T_{w}, w \in \tilde{W}_{L}$, and relations

$$
T_{w} T_{w^{\prime}}=T_{w w^{\prime}}, \quad \text { if } \ell\left(w w^{\prime}\right)=\ell(w)+\ell\left(w^{\prime}\right) .
$$

Let $\tilde{\mathcal{B}}_{\text {aff }}=\tilde{\mathcal{B}}_{Q}$. View $L / Q$ as a subgroup of $\Omega \cong P / Q$. Then $\mathcal{B}_{L}=\mathcal{B}_{\text {aff }} \rtimes L / Q$ is presented by generators $T_{i}=T_{s_{i}}, 0 \leq i \leq n$, and relations

$$
\underbrace{T_{i} T_{j} \cdots}_{m_{i j}}=\underbrace{T_{j} T_{i} \cdots}_{m_{i j}}, \quad \text { and } \quad g T_{i} g^{-1}=T_{\sigma(i)}, \quad \text { for } g \in \Omega,
$$

where $\sigma$ is as in (4.4), and the $m_{i j}$ are specified by the extended Dynkin diagram of $R^{\vee}$.

Let $P^{+}=\sum_{i=1}^{n} \mathbb{Z}_{\geq 0} \omega_{i}$ be the dominant weights in $P$. Define elements $X^{\lambda}, \lambda \in P$ by

$$
X^{\lambda}=T_{t_{\lambda}}, \quad \text { if } \lambda \in P^{+} \text {, and } \quad X^{\lambda}=X^{\mu}\left(X^{\nu}\right)^{-1}, \quad \text { if } \lambda=\mu-\nu \text { with } \mu, \nu \in P^{+} .
$$

By $[\mathrm{Mac} 3,3.4]$ and $[\mathrm{Lu}]$, the $X^{\lambda}$ are well defined and do not depend on the choice $\lambda=\mu-\nu$, and

$$
X^{\lambda} X^{\mu}=X^{\mu} X^{\lambda}=X^{\lambda+\mu}, \quad \text { for } \lambda, \mu \in P .
$$

Then $X^{\lambda} \in \tilde{\mathcal{B}}_{L}$ if and only if $\lambda \in L$.

(4.9) Affine Hecke algebras. Fix $q \in \mathbb{C}^{*}$. The affine Hecke algebra $\widetilde{H}_{L}$ is the quotient of the group algebra $\mathbb{C} \tilde{\mathcal{B}}_{L}$ by the relations

$$
\left(T_{i}-q\right)\left(T_{i}+q^{-1}\right)=0, \quad \text { for } 0 \leq i \leq n .
$$

In $\tilde{H}_{L}($ see $[\mathrm{Mac}, 4.2])$,

$$
X^{\lambda} T_{i}=T_{i} X^{s_{i} \lambda}+\left(q-q^{-1}\right) \frac{X^{\lambda}-X^{s_{i} \lambda}}{1-X^{-\alpha_{i}}}, \quad \text { for } \lambda \in L, 1 \leq i \leq n .
$$


The Iwahori-Hecke algebra $H$ is the subalgebra of $\tilde{H}$ generated by $T_{1}, \ldots, T_{n}$.

To our knowledge, the following theorem is due to Bernstein and Zelevinsky in type A, and to Bernstein in general type (unpublished). Lusztig has given an exposition in [Lu]. We give a new proof which we believe is more elementary and more direct.

Theorem 4.12. (Bernstein, Zelevinsky, Lusztig $[L u]$ ) Let $L$ be a lattice such that $Q \subseteq L \subseteq P$, where $Q$ is the root lattice and $P$ is the weight lattice of the root system $R$. Let $\tilde{H}=\overline{\tilde{H}}_{L}$ be the affine Hecke algebra corresponding to $L$ and let $\mathbb{C}[X]=\operatorname{span}\left\{X^{\lambda} \mid \lambda \in L\right\}$. Let $W$ be the Weyl group of $R$. Then the center of $\tilde{H}$ is

$$
Z(\tilde{H})=\mathbb{C}[X]^{W}=\{f \in \mathbb{C}[X] \mid w f=f \text { for every } w \in W\}
$$

Proof. Assume

$$
z=\sum_{\lambda \in L, w \in W} c_{\lambda, w} X^{\lambda} T_{w} \in Z(\tilde{H})
$$

Let $m \in W$ be maximal in Bruhat order subject to $c_{\gamma, m} \neq 0$ for some $\gamma \in L$. If $m \neq 1$ there exists a dominant $\mu \in L$ such that $c_{\gamma+\mu-m \mu, m}=0$ (otherwise $c_{\gamma+\mu-m \mu, m} \neq 0$ for every dominant $\mu \in L$, which is impossible since $z$ is a finite linear combination of $\left.X^{\lambda} T_{w}\right)$. Since $z \in Z(\tilde{H})$ we have

$$
z=X^{-\mu} z X^{\mu}=\sum_{\lambda \in L, w \in W} c_{\lambda, w} X^{\lambda-\mu} T_{w} X^{\mu}
$$

Repeated use of the relation (4.11) yields

$$
T_{w} X^{\mu}=\sum_{\nu \in L, v \in W} d_{\nu, v} X^{\nu} T_{v}
$$

where $d_{\nu, v}$ are constants such that $d_{w \mu, w}=1, d_{\nu, w}=0$ for $\nu \neq w \mu$, and $d_{\nu, v}=0$ unless $v \leq w$. So

$$
z=\sum_{\lambda \in L, w \in W} c_{\lambda, w} X^{\lambda} T_{w}=\sum_{\lambda \in L, w \in W} \sum_{\nu \in L, v \in W} c_{\lambda, w} d_{\nu, v} X^{\lambda-\mu+\nu} T_{v}
$$

and comparing the coefficients of $X^{\gamma} T_{m}$ gives $c_{\gamma, m}=c_{\gamma+\mu-m \mu, m} d_{m \mu, m}$. Since $c_{\gamma+\mu-m \mu, m}=0$ it follows that $c_{\gamma, m}=0$, which is a contradiction. Hence $z=\sum_{\lambda \in L} c_{\lambda} X^{\lambda} \in \mathbb{C}[X]$.

The relation (4.11) gives

$$
z T_{i}=T_{i} z=\left(s_{i} z\right) T_{i}+\left(q-q^{-1}\right) z^{\prime}
$$

where $z^{\prime} \in \mathbb{C}[X]$. Comparing coefficients of $X^{\lambda}$ on both sides yields $z^{\prime}=0$. Hence $z T_{i}=\left(s_{i} z\right) T_{i}$, and therefore $z=s_{i} z$ for $1 \leq i \leq n$. So $z \in \mathbb{C}[X]^{W}$.

(4.13) Deducing the $\tilde{H}_{L}$ representation theory from $\tilde{H}_{P}$. Although we have not taken this point of view in our presentation, the affine Hecke algebras defined above are naturally associated to a reductive algebraic group $G$ over $\mathbb{C}[\mathrm{KL}]$ or a $p$-adic Chevalley group $[\mathrm{IM}]$. In this formulation, the lattice $L$ is determined by the group of characters of the maximal torus of $G$. It is often convenient to work only with the adjoint version or only with the simply connected version of the group $G$ and therefore it seems desirable to be able to derive the representation theory of the affine Hecke algebras $\tilde{H}_{L}$ from the representation theory of the affine Hecke algebra $\tilde{H}_{P}$. The following 
theorem shows that this can be done in a simple way by using the extension of Clifford theory in the Appendix. In particular, Theorem A.13, can be used to construct all of the simple $\tilde{H}_{L}$-modules from the simple $\tilde{H}_{P}$-modules.

Theorem 4.14. Let $L$ be a lattice such that $Q \subseteq L \subseteq P$, where $Q$ is the root lattice and $P$ is the weight lattice of the root system $R$. Let $\tilde{H}=\tilde{H}_{L}$ be the affine Hecke algebra corresponding to $L$. Then there is an action of a finite group $K$ on $\tilde{H}_{P}$, acting by automorphisms, such that

$$
\tilde{H}_{L}=\left(\tilde{H}_{P}\right)^{K},
$$

is the subalgebra of fixed points under the action of the group $K$.

Proof. There are two cases to consider, depending on whether the group $\Omega \cong P / Q$ is cyclic or not.

$$
\begin{array}{ccccccccccc}
\text { Type } & A_{n-1} & B_{n} & C_{n} & D_{2 n-1} & D_{2 n} & E_{6} & E_{7} & E_{8} & F_{4} & G_{2} \\
\Omega & \mathbb{Z} / n \mathbb{Z} & \mathbb{Z} / 2 \mathbb{Z} & \mathbb{Z} / 2 \mathbb{Z} & \mathbb{Z} / 4 \mathbb{Z} & \mathbb{Z} / 2 \mathbb{Z} \times \mathbb{Z} / 2 \mathbb{Z} & \mathbb{Z} / 3 \mathbb{Z} & \mathbb{Z} / 2 \mathbb{Z} & 1 & 1 & 1
\end{array}
$$

In each case we construct the group $K$ and its action on $\tilde{H}_{P}$ explicitly. This is necessary for the effective application of Theorem A.13 on examples.

Case 1. If $\Omega$ is a cyclic group $\Omega$ then the subgroup $L / Q$ is a cyclic subgroup. Suppose

$$
\Omega=\left\{1, g, \ldots, g^{r-1}\right\} \quad \text { and } \quad L / Q=\left\{1, g^{d}, \ldots, g^{d(p-1)}\right\},
$$

where $p d=r$. Let $\zeta$ be a primitive $p$ th root of unity and define an automorphism

$$
\begin{aligned}
\sigma: \tilde{H}_{P} & \longmapsto \tilde{H}_{P} \\
g & \longmapsto \zeta g, \\
T_{i} & \longmapsto T_{i}, \quad 0 \leq i \leq n .
\end{aligned}
$$

The map $\sigma$ is an algebra isomorphism since it preserves the relations in (4.6) and (4.10). Furthermore, $\sigma$ gives rise to a $\mathbb{Z} / p \mathbb{Z}$ action on $\tilde{H}_{P}$ and

$$
\tilde{H}_{L}=\left(\tilde{H}_{P}\right)^{\mathbb{Z} / p \mathbb{Z}} .
$$

Case 2. If the root system $R^{\vee}$ is of type $D_{n}, n$ even, then $\Omega \cong \mathbb{Z} / 2 \mathbb{Z} \times \mathbb{Z} / 2 \mathbb{Z}$ and the subgroups of $\Omega$ correspond to the intermediate lattices $Q \subseteq L \subseteq P$. Suppose

$$
\Omega=\left\{1, g_{1}, g_{2}, g_{1} g_{2} \mid g_{1}^{2}=g_{2}^{2}=1, g_{1} g_{2}=g_{2} g_{1}\right\} .
$$

and define automorphisms of $\tilde{H}_{P}$ by

$$
\begin{aligned}
\sigma_{1}: \tilde{H}_{P} & \longmapsto \tilde{H}_{P} \\
g_{1} & \longmapsto-g_{1}, \\
g_{2} & \longmapsto g_{2}, \\
T_{i} & \longmapsto T_{i},
\end{aligned}
$$

and

$\begin{aligned} & \sigma_{2}: \tilde{H}_{P} \longmapsto \tilde{H}_{P} \\ & g_{1} \longmapsto g_{1}, \\ & g_{2} \longmapsto-g_{2}, \\ & T_{i} \longmapsto \\ & T_{i} .\end{aligned}$

Then

$$
\tilde{H}_{L_{1}}=\left(\tilde{H}_{P}\right)^{\sigma_{1}}, \quad \tilde{H}_{L_{2}}=\left(\tilde{H}_{P}\right)^{\sigma_{2}}, \quad \text { and } \quad \tilde{H}_{Q}=\left(\tilde{H}_{P}\right)^{\left\langle\sigma_{1}, \sigma_{2}\right\rangle},
$$

where $L_{1}$ and $L_{2}$ are the two intermediate lattices strictly between $Q$ and $P$.

\section{Where DOES THE HOMOMORPHISM $\Phi$ COME FROM?}


The homomorphism $\Phi: H_{\infty, 1, n} \rightarrow H_{r, 1, n}$ of Proposition 2.5 is a powerful tool for transporting results about the affine Hecke algebra of type $A$ to the cyclotomic Hecke algebras. In this section we show how this homomorphism arises naturally, from a folding of the Dynkin diagram of $\tilde{B}_{n}$, and we give some generalizations of the homomorphism $\Phi$ to other types.

Example 1. Type $C_{n}$. The root system $R$ of type $C_{n}$ can be realized by

$$
R=\left\{ \pm 2 \varepsilon_{i}, \pm\left(\varepsilon_{j}-\varepsilon_{i}\right) \mid 1 \leq i, j \leq n\right\}
$$

where $\varepsilon_{i}$ are an orthonormal basis of $\mathbb{R}^{n}$. The simple roots and the fundamental weights are given by

$$
\begin{aligned}
& \alpha_{1}=2 \varepsilon_{1}, \quad \alpha_{i}=\varepsilon_{i}-\varepsilon_{i-1}, \quad 2 \leq i \leq n, \\
& \omega_{i}=\varepsilon_{n}+\varepsilon_{n-1}+\cdots+\varepsilon_{i}, \quad 1 \leq i \leq n .
\end{aligned}
$$

If $\phi^{\vee}$ is the highest root of $R^{\vee}$ then

$$
\phi=\varepsilon_{n}+\varepsilon_{n-1}, \quad \text { and } \quad s_{\phi^{\vee}}=\left(s_{n-1} \cdots s_{2} s_{1} s_{2} \cdots s_{n-1}\right) s_{n}\left(s_{n-1} \cdots s_{2} s_{1} s_{2} \cdots s_{n-1}\right) .
$$

Then $\omega_{n}=\varepsilon_{n}$ is the only miniscule weight,

$$
\begin{aligned}
w_{0} & =(1,-1)(2,-2) \cdots(n,-n), \\
w_{0, n} & =(1,-1)(2,-2) \cdots(n-1,-(n-1)), \quad \text { and } \\
w_{0} w_{0, n} & =(n,-n)=s_{n} \cdots s_{2} s_{1} s_{2} \cdots s_{n} .
\end{aligned}
$$

Thus, from (4.2), (4.3) and (4.6), $\Omega=\left\{1, g_{n}\right\} \cong \mathbb{Z} / 2 \mathbb{Z}$,

$$
\begin{aligned}
& g_{n}=X^{\varepsilon_{n}} T_{n}^{-1} \cdots T_{2}^{-1} T_{1}^{-1} T_{2}^{-1} \cdots T_{n}^{-1}, \\
& T_{0}=X^{\varepsilon_{n}+\varepsilon_{n-1}}\left(T_{n}^{-1} \cdots T_{2}^{-1} T_{1}^{-1} T_{2}^{-1} \cdots T_{n}^{-1}\right) T_{n}^{-1}\left(T_{n}^{-1} \cdots T_{2}^{-1} T_{1}^{-1} T_{2}^{-1} \cdots T_{n}^{-1}\right),
\end{aligned}
$$

and

$$
g_{n} T_{0} g_{n}^{-1}=T_{n}, \quad \text { and } \quad g_{n} T_{n} g_{n}^{-1}=T_{0} .
$$

The braid group $\tilde{\mathcal{B}}_{P}\left(C_{n}\right)$ is generated by $T_{0}, T_{1}, \ldots, T_{n}$ and $g_{n}$ which satisfy relations in (4.6), where the $m_{i j}$ are given by the extended Dynkin diagram $\tilde{B}_{n}$, see Figure 3 . The braid group $\mathcal{B}\left(C_{n}\right)$ is the subgroup generated by $T_{1}, \ldots, T_{n}$. These elements satisfy the relations in (4.6), where the $m_{i j}$ are given by the Dynkin diagram $C_{n}$. A straightforward check verifies that the map defined by
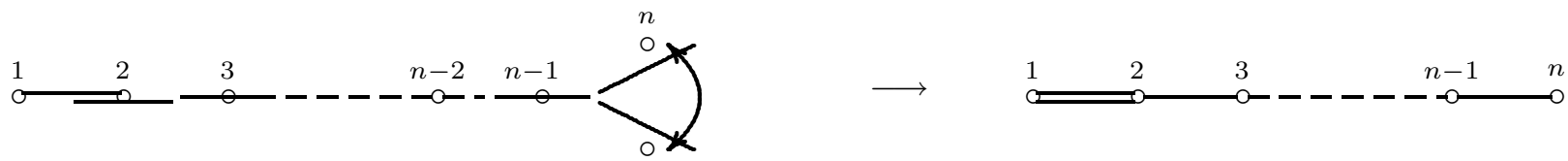

0

$$
\begin{aligned}
& \Phi_{\tilde{C} C}: \quad \tilde{\mathcal{B}}_{P}\left(B_{n}\right) \quad \longrightarrow \mathcal{B}\left(C_{n}\right) \\
& g_{n} \quad \longmapsto \quad 1 \text {, } \\
& T_{0} \quad \longmapsto \quad T_{n}, \\
& T_{i} \quad \longmapsto \quad T_{i}, \quad 1 \leq i \leq n .
\end{aligned}
$$


extends to a well defined surjective group homomorphism. From the identity (4.3),

$$
\begin{aligned}
\Phi_{\tilde{C} C}\left(X^{\varepsilon_{n}}\right) & =\Phi_{\tilde{C} C}\left(X^{\omega_{n}}\right) \\
& =\Phi_{\tilde{C} C}\left(g_{n} T_{n} T_{n-1} \cdots T_{2} T_{1} T_{2} \cdots T_{n-1} T_{n}\right) \\
& =T_{n} T_{n-1} \cdots T_{2} T_{1} T_{2} \cdots T_{n-1} T_{n} .
\end{aligned}
$$

By inductively applying the relation $X^{\varepsilon_{i}}=T_{i} X^{\varepsilon_{i-1}} T_{i}$ we get

$$
\Phi_{\tilde{C} C}\left(X^{\varepsilon_{i}}\right)=T_{i} T_{i-1} \cdots T_{2} T_{1} T_{2} \cdots T_{i-1} T_{i}, \quad \text { for all } 1 \leq i \leq n .
$$

Example 2. Type $A_{n-1}$. Since the weight lattice $P$ for the root system of type $C_{n}$ is the same as the lattice $L$ defined in (1.5) we have an injective homomorphism

$$
\begin{aligned}
\Phi_{\tilde{A} \tilde{C}}: \mathcal{B}_{\infty, 1, n} & \longrightarrow \tilde{\mathcal{B}}_{P}\left(C_{n}\right) \\
T_{i} & \longmapsto T_{i}, \\
X^{\varepsilon_{i}} & \longmapsto X^{\varepsilon_{i}} .
\end{aligned}
$$

The composition of $\Phi_{\tilde{A} \tilde{C}}$ and the map $\Phi_{\tilde{C} C}$ from (5.5) is the surjective homomorphism defined by

$$
\begin{array}{rlrl}
\Phi: \mathcal{B}_{\infty, 1, n} & \longmapsto \mathcal{B}\left(C_{n}\right) & \\
T_{i} & \longmapsto T_{i}, & & 2 \leq i \leq n, \\
X^{\varepsilon_{i}} & \longmapsto T_{i} T_{i-1} \cdots T_{2} T_{1} T_{2} \cdots T_{i-1} T_{i}, & & 1 \leq i \leq n .
\end{array}
$$

In fact, it follows from the defining relations of $\mathcal{B}_{\infty, 1, n}$ and $\mathcal{B}\left(C_{n}\right)$ that the map $\Phi$ is an isomorphism!

The cyclotomic Hecke algebras $H_{r, 1, n}\left(u_{1}, \ldots, u_{r} ; q\right)$ are quotients of $\mathbb{C B}\left(C_{n}\right)$ and in this way the group homomorphism $\Phi$ is the source of the algebra homomorphism

$$
\Phi: H_{\infty, 1, n} \longrightarrow H_{r, 1, n}\left(u_{1}, \ldots, u_{r} ; q\right)
$$

which was used extensively in Section 3 to relate the representation theory of the cyclotomic Hecke algebras $H_{r, 1, n}\left(u_{1}, \ldots, u_{r} ; q\right)$ to the affine Hecke algebra of type A.

Example 3. Type $D_{n}$. Let $R$ be the root system of type $D_{n}$. Then $R^{\vee}$ is also of type $D_{n}$ and inspection of the Dynkin diagrams of types $\tilde{D}_{n}$ and $D_{n}$ yields a surjective algebra homomorphism defined by

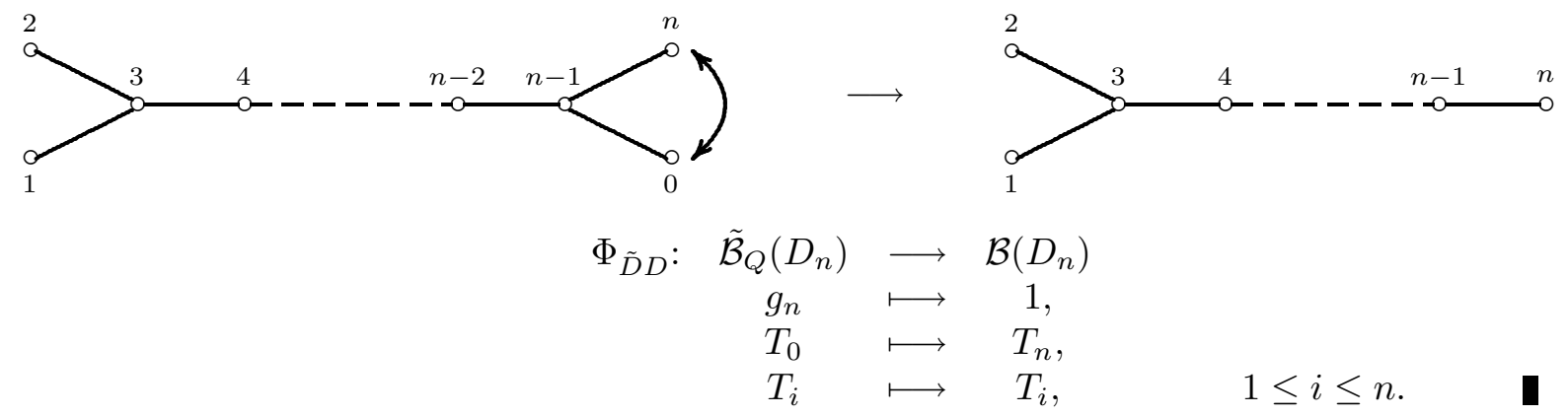

Examples 1, 2, and 3 show that, for types $A, B$ and $D$, there exist surjective homomorphisms from the affine Hecke algebra to the corresponding Iwahori-Hecke subalgebra. The following example shows that this is not a general phenomenon: there does not exist a surjective algebra 
homomorphism from the affine Hecke algebra of type $G_{2}$ to the corresponding Iwahori-Hecke subalgebra of type $G_{2}$.

Example 4. Type $G_{2}$. If $R$ is the root system of type $G_{2}$ then $P=Q$ and $\Omega=\{1\}$.

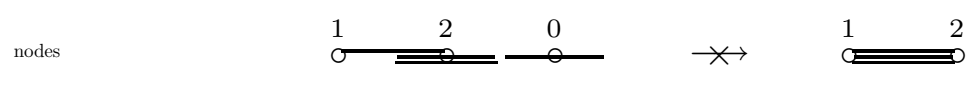

Proposition 5.6. Let $\tilde{H}\left(G_{2}\right)$ be the affine Hecke algebra of type $G_{2}$ as given by (4.6) and (4.10) and let $H\left(G_{2}\right)$ be the Iwahori-Hecke subalgebra of type $G_{2}$ generated by $T_{1}$ and $T_{2}$. There does not exist an algebra homomorphism $\Phi: \tilde{H}\left(G_{2}\right) \rightarrow H\left(G_{2}\right)$ such that $\Phi\left(T_{i}\right)=T_{i}$, for $1 \leq i \leq 2$.

Proof. There is an irreducible representation of $\tilde{H}\left(G_{2}\right)$ given by

$$
\rho\left(T_{1}\right)=\left(\begin{array}{cc}
q & 0 \\
0 & -q^{-1}
\end{array}\right), \quad \text { and } \quad \rho\left(T_{2}\right)=\frac{1}{q+q^{-1}}\left(\begin{array}{cc}
2-q^{-2} & q^{2}-1+q^{-2} \\
3 & q^{2}-2
\end{array}\right)
$$

(see [Ra1, Theorem 6.11]). We show that there does not exist a $2 \times 2$ matrix $N$ which satisfies

$$
N^{2}=\left(q-q^{-1}\right) N+1, \quad N \rho\left(T_{2}\right) N=\rho\left(T_{2}\right) N \rho\left(T_{2}\right) \quad \text { and } \quad N \rho\left(T_{1}\right)=\rho\left(T_{1}\right) N .
$$

If $N$ exists then $N$ must be diagonal since $N$ commutes with $\rho\left(T_{1}\right)$ and $\rho\left(T_{1}\right)$ is a diagonal matrix with distinct eigenvalues. The first equation shows that $N$ is invertible and the second equation shows that $N$ is conjugate to $\rho\left(T_{2}\right)$. It follows that $N$ must have one eigenvalue $q$ and one eigenvalue $-q^{-1}$. Thus, either

$$
N=\left(\begin{array}{cc}
q & 0 \\
0 & -q^{-1}
\end{array}\right) \quad \text { or } \quad N=\left(\begin{array}{cc}
-q^{-1} & 0 \\
0 & q
\end{array}\right) .
$$

However, neither of these matrices satisfies the relation $N \rho\left(T_{2}\right) N=\rho\left(T_{2}\right) N \rho\left(T_{2}\right)$. This contradiction shows that the representation $\rho$ cannot be extended to be a representation of $\tilde{H}\left(G_{2}\right)$.

In spite of the fact, demonstrated by the previous example, that there does not always exist a surjective algebra homomorphism from the affine Hecke algebra onto its Iwahori-Hecke subalgebra, there are interesting surjective homomorphisms from affine Hecke algebras of exceptional type.

Example 5. Type $E_{6}$. For the root system of type $E_{6}, P / Q \cong \mathbb{Z} / 3 \mathbb{Z}$. Let $\Omega=\left\{1, g, g^{2}\right\}$ where $g$ is as given by (4.3) for the minuscule weight $\omega_{1}$ (see [Bou, p. 261]). There are surjective algebra homomorphisms
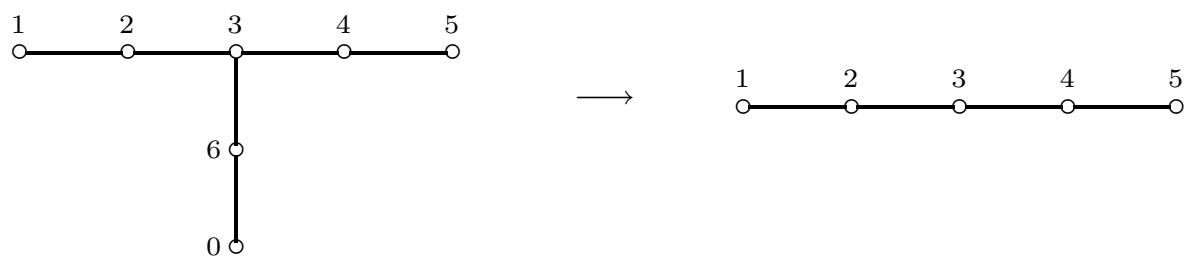

$$
\begin{aligned}
& \Phi: \quad \tilde{\mathcal{B}}_{Q}\left(E_{6}\right) \quad \longrightarrow \mathcal{B}\left(A_{5}\right) \\
& T_{0} \longmapsto T_{5}, \\
& T_{6} \longmapsto T_{4}, \\
& T_{i} \quad \longmapsto \quad T_{i}, \quad 1 \leq i \leq 5,
\end{aligned}
$$


and
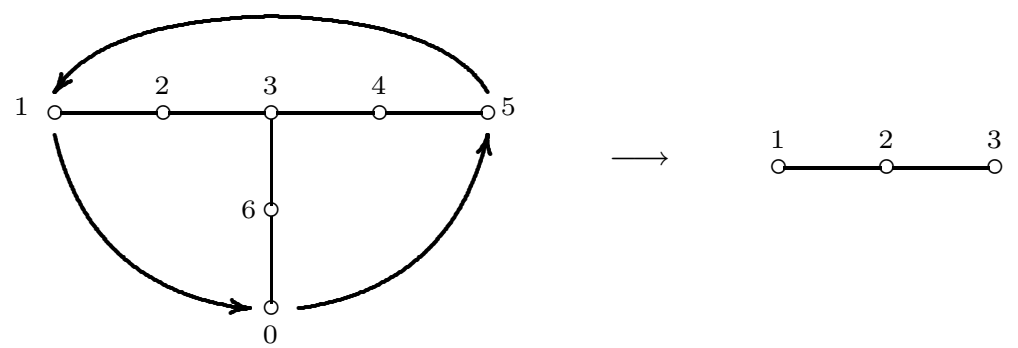

$$
\begin{aligned}
& \Phi^{\prime}: \quad \tilde{\mathcal{B}}_{P}\left(E_{6}\right) \quad \longrightarrow \mathcal{B}\left(A_{3}\right) \\
& g \quad \longmapsto \quad 1 \text {, } \\
& T_{0} \quad \longmapsto \quad T_{1} \text {, } \\
& T_{6} \quad \longmapsto \quad T_{2} \text {, } \\
& T_{5} \longmapsto T_{1}, \\
& T_{4} \longmapsto T_{2} \text {, } \\
& T_{i} \quad \longmapsto \quad T_{i}, \quad 1 \leq i \leq 3 .
\end{aligned}
$$

(5.7) Example 6. Type $F_{4}$. For the root system $R$ of type $F_{4}$ we have $P=Q$ and $\Omega=\{1\}$. There is a surjective homomorphism
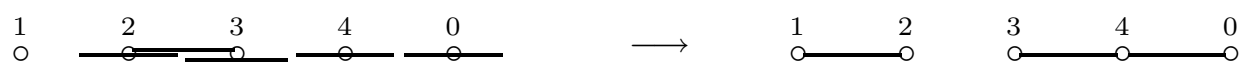

$\Phi: \quad \tilde{\mathcal{B}}\left(F_{4}\right) \quad \longrightarrow \mathcal{B}\left(A_{2} \times A_{3}\right)$
$T_{i} \longmapsto T_{i}$,
$0 \leq i \leq 4$

\section{Appendix: Clifford Theory}

Let $R$ be an algebra over $\mathbb{C}$ and let $G$ be a finite group acting by automorphisms on $R$. The skew group ring is

$$
R \rtimes G=\left\{\sum_{g \in G} r_{g} g \mid r_{g} \in R\right\}
$$

with multiplication given by the distributive law and the relation

$$
g r=g(r) g, \quad \text { for } g \in G \text { and } r \in R .
$$

Let $N$ be a (finite dimensional) left $R$-module. For each $g \in G$ define an $R$-module ${ }^{g} N$, which has the same underlying vector space $N$ but such that

$$
{ }^{g} N \text { has } R \text {-action given by } \quad r \circ n=g^{-1}(r) n \text {, }
$$

for $r \in R, n \in N$. If $W$ is an $R$-submodule of $N$ then ${ }^{g} W$ is an $R$-submodule of ${ }^{g} N$ and so ${ }^{g} N$ is simple if and only if $N$ is simple. Thus there is an action of $G$ on the set of simple $R$-modules.

Let $R^{\lambda}$ be a simple $R$-module. The inertia group of $R^{\lambda}$ is

$$
H=\left\{h \in G \mid R^{\lambda} \cong{ }^{h} R^{\lambda}\right\} .
$$


If $h \in H$ then Schur's lemma implies that the isomorphism $R^{\lambda} \cong{ }^{h} R^{\lambda}$ is unique up to constant multiples (since both $R^{\lambda}$ and ${ }^{h} R^{\lambda}$ are simple). For each $h \in H$ fix an isomorphism $\phi_{h}: R^{\lambda} \rightarrow h^{-1} R^{\lambda}$. Then, as operators on $R^{\lambda}$,

$$
\phi_{h} r=h(r) \phi_{h}, \quad \text { and } \quad \phi_{g} \phi_{h}=\alpha(g, h) \phi_{g h},
$$

where $\alpha(g, h) \in \mathbb{C}^{*}$ are determined by the choice of the isomorphisms $\phi_{h}$. The resulting function $\alpha: H \times H \rightarrow \mathbb{C}^{*}$ is called a factor set $[\mathrm{CR}, 8.32]$.

Let $(\mathbb{C} H)_{\alpha^{-1}}$ be the algebra with basis $\left\{c_{h} \mid h \in H\right\}$ and multiplication given by

$$
c_{g} c_{h}=\alpha(g, h)^{-1} c_{g h}, \quad \text { for } g, h \in H .
$$

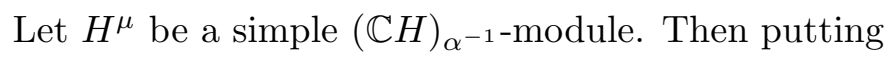

$$
r h(m \otimes n)=r \phi_{h} m \otimes c_{h} n, \quad \text { for } r \in R, h \in H, m \in R^{\lambda}, n \in H^{\mu},
$$

defines an action of $R \rtimes H$ on $R^{\lambda} \otimes H^{\mu}$.

Theorem A.6. (Clifford theory) Let $R^{\lambda}$ be a simple $R$-module and let $H$ be the inertia group of

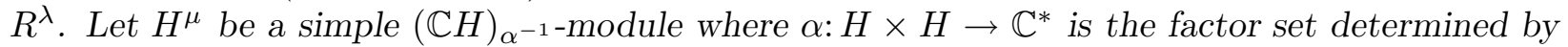
a choice of isomorphisms $\phi_{h}: R^{\lambda} \rightarrow{ }^{h} R^{\lambda}$. Define an action of $R \rtimes H$ on $R^{\lambda} \otimes H^{\mu}$ as in (A.5) and define

$$
R G^{\lambda, \mu}=\operatorname{Ind}_{R \rtimes H}^{R \rtimes G}\left(R^{\lambda} \otimes H^{\mu}\right)=(R \rtimes G) \otimes_{R \rtimes H}\left(R^{\lambda} \otimes H^{\mu}\right) .
$$

Then

(a) $R G^{\lambda, \mu}$ is a simple $R \rtimes G$-module.

(b) Every simple $R \rtimes G$-module is obtained by this construction.

(c) If $R G^{\lambda, \mu} \cong R G^{\nu, \gamma}$ then $R^{\lambda}$ and $R^{\nu}$ are in the same $G$-orbit of simple $R$-modules and $H^{\mu} \cong H^{\gamma}$ as $(\mathbb{C} H)_{\alpha^{-1} \text {-modules. }}$

Proof. The proof of this theorem is as in [Mac2] except that the consideration of the factor set $\alpha: H \times H \rightarrow \mathbb{C}^{*}$ is necessary to correct an error there. We thank P. Deligne for pointing this out to us. A sketch of the proof is as follows.

Let $M$ be a simple $R \rtimes G$-module and let $R^{\lambda}$ be a simple $R$-submodule of $M$. Then $g R^{\lambda} \cong{ }^{g} R^{\lambda}$ as $R$-modules and $M=\sum_{g \in G} g R^{\lambda}$ since the right hand side is an $R \rtimes G$-submodule of $M$. Then

$$
M=\sum_{g_{i} \in G / H} g_{i} N=\operatorname{Ind}_{R \rtimes H}^{R \rtimes G}(N), \quad \text { where } \quad N=\sum_{h \in H} h R^{\lambda},
$$

and the first sum is over a set $\left\{g_{i}\right\}$ of coset representatives of the cosets $G / H$.

The $R$-module $N$ is semisimple and by [Bou2]

$$
N \cong R^{\lambda} \otimes H^{\mu}
$$

where $H^{\mu}=\operatorname{Hom}_{R}\left(R^{\lambda}, N\right)$. It can be checked that the vector space $H^{\mu}$ has a $(\mathbb{C} H)_{\alpha^{-1} \text {-action }}$ given by

$$
\left(c_{h} \psi\right)(m)=\alpha\left(h, h^{-1}\right) h \psi\left(\phi_{h^{-1}}(m)\right), \quad \text { for } h \in H, \psi \in \operatorname{Hom}_{R}\left(R^{\lambda}, N\right),
$$

where $c_{h}$ is as in (A.4). Then, with $R \rtimes H$-action on $R^{\lambda} \otimes H^{\mu}$ given by (A.5), the isomorphism in (A.7) is an isomorphism of $R \rtimes H$-modules (see [CR, Thm. (11.17) (ii)]). 


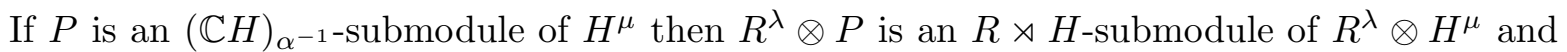
$\operatorname{Ind}_{R \rtimes H}^{R \rtimes G}\left(R^{\lambda} \otimes P\right)$ is an $R \rtimes G$-submodule of $M$. Thus $H^{\mu}$ must be a simple $(\mathbb{C} H)_{\alpha^{-1}}$-module.

This argument shows that every simple $R \rtimes G$-module is of the form $R G^{\lambda, \mu}$. The uniqueness follows as in [Mac2, App.].

Remark A.8. A different choice $\psi_{h}: R^{\lambda} \rightarrow{ }^{h} R^{\lambda}$ of the isomorphisms in (A.3) may yield a factor set $\beta: H \times H \rightarrow \mathbb{C}^{*}$ which is different from the factor set $\alpha$. However, the algebras $(\mathbb{C} H)_{\beta^{-1}}$ and $(\mathbb{C} H)_{\alpha^{-1}}$ are always isomorphic (a diagonal change of basis suffices).

Lemma A.9. Define $R^{G}=\{r \in R \mid g(r)=r$ for all $g \in G\}$ and let $e=(1 /|G|) \sum_{g \in G} g \in R \rtimes G$.

(a) The map

$$
\begin{aligned}
\theta: \quad R^{G} & \longrightarrow e(R \rtimes G) e \\
s & \longmapsto s e
\end{aligned}
$$

is a ring isomorphism.

(b) Left multiplication by elements of $R$ and the action of $G$ by automorphisms make $R$ into a left $R \rtimes G$-module. Right multiplication makes $R$ a right $R^{G}$-module. The rings $R \rtimes G$ and $e(R \rtimes G) e$ act on $(R \rtimes G) e$ by left and right multiplication, respectively. The map

$$
\begin{array}{rlc}
\psi: R & \cong(R \rtimes G) e \\
r & \longmapsto r e
\end{array}
$$

is an isomorphism of $\left(R \rtimes G, R^{G}\right)$-bimodules.

Proof. (a) If $r \in R^{G}$ then

$$
\text { ere }=\frac{1}{|G|} \sum_{g \in G} g(r) g e=\frac{1}{|G|} \sum_{g \in G} r e=r e .
$$

Thus the map $\theta$ is well defined and if $r, s \in R^{G}$ then rese $=r s e$, so $\theta$ is a homomorphism. If $r e=s e$ then $r=s$ since $R \rtimes G$ is a free $R$-module with basis $G$. Thus $\theta$ is injective. If $\sum_{g \in G} r_{g} g$ is a general element of $R \rtimes G$ then

$$
e\left(\sum_{g \in G} r_{g} g\right) e=\sum_{g, h \in G} h\left(r_{g}\right) h g e=\left(\sum_{g, h \in G} h\left(r_{g}\right)\right) e
$$

and, for each $g \in G, \sum_{h \in G} h\left(r_{g}\right) \in R^{G}$. So $\theta$ is surjective.

The proof of (b) is straightforward.

Let $(\mathbb{C} H)_{\alpha}$ be the algebra with basis $\left\{b_{h} \mid h \in H\right\}$ and multiplication given by

$$
b_{g} b_{h}=\alpha(g, h) b_{g h}, \quad \text { for } g, h \in H
$$

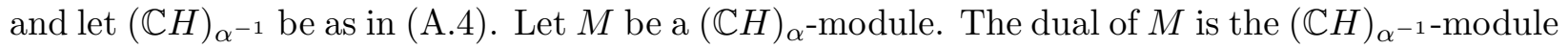
given by the vector space $M^{*}=\operatorname{Hom}(M, \mathbb{C})$ with action

$$
\left(c_{h} \psi\right)(m)=\alpha\left(h, h^{-1}\right)^{-1} \psi\left(b_{h^{-1}} m\right), \quad \text { for } h \in H, \psi \in M^{*} .
$$


This is a $(\mathbb{C} H)_{\alpha^{-1}}$ action since, for all $g, h \in H, \psi \in M^{*}$,

$$
\begin{aligned}
\left(c_{g} c_{h} \psi\right)(m) & =\alpha\left(h, h^{-1}\right)^{-1} \alpha\left(g, g^{-1}\right)^{-1} \psi\left(b_{h^{-1}} b_{g^{-1}} m\right) \\
& =\alpha\left(h, h^{-1}\right)^{-1} \alpha\left(g, g^{-1}\right)^{-1} \alpha\left(h^{-1}, g^{-1}\right) \psi\left(b_{(g h)^{-1}} m\right) \\
& =\alpha\left(h, h^{-1}\right)^{-1} \alpha\left(g, g^{-1}\right)^{-1} \alpha\left(h^{-1}, g^{-1}\right) \alpha\left(g h, h^{-1} g^{-1}\right)\left(c_{g h} \psi\right)(m) \\
& =\alpha(g, h)^{-1}\left(c_{g h} \psi\right)(m),
\end{aligned}
$$

where the last equality follows from the associativity of the product $b_{g} b_{h} b_{h^{-1}} b_{g^{-1}}$ in $(\mathbb{C} H)_{\alpha}$. If $\rho:(\mathbb{C} H)_{\alpha} \rightarrow \operatorname{End}(M)$ is the representation corresponding to $M$ then the representation $\rho^{*}:(\mathbb{C} H)_{\alpha^{-1}} \rightarrow$ $\operatorname{End}\left(M^{*}\right)$ corresponding to $M^{*}$ is

$$
\rho^{*}\left(c_{h}\right)=\alpha\left(h, h^{-1}\right)^{-1} \rho\left(b_{h^{-1}}\right)^{t}=\left(\rho\left(b_{h}\right)^{-1}\right)^{t} .
$$

If $M$ is a $(\mathbb{C} H)_{\alpha}$-module and $N$ is a $(\mathbb{C} H)_{\alpha^{-1}}$-module then $M \otimes N$ is an $\mathbb{C} H$-module with action defined by

$$
h(m \otimes n)=b_{h} m \otimes c_{h} n, \quad \text { for } h \in H, m \in M \text { and } n \in N .
$$

The following lemma is a version of Schur's lemma which will be used in the proof of Theorem A.13.

Lemma A.12. Suppose that $M$ and $N$ are simple $(\mathbb{C} H)_{\alpha}$-modules and let $N^{*}$ be the $(\mathbb{C} H)_{\alpha^{-1-}}$ module which is the dual of $N$. Let $e_{H}=(1 /|H|) \sum_{h \in H} h$. Then

$$
\operatorname{dim}\left(e_{H}\left(M \otimes N^{*}\right)\right)= \begin{cases}1, & \text { if } M \cong N \\ 0, & \text { otherwise }\end{cases}
$$

Proof. Identify $M \otimes N^{*}$ with $\operatorname{Hom}(N, M)$. Then, by (A.10) and (A.11), the action of $\mathbb{C} H$ on $\operatorname{Hom}(N, M)$ is given by,

$$
h A=\rho\left(b_{h}\right) A \rho\left(b_{h}\right)^{-1}, \quad \text { for } h \in H \text { and } A \in \operatorname{Hom}(N, M),
$$

where $\rho:(\mathbb{C} H)_{\alpha} \rightarrow \operatorname{End}(M)$ is the representation corresponding to $M$. If $A \in \operatorname{Hom}(N, M)$ and $g \in H$ then

$$
e_{H} A=g e_{H} A=g\left(e_{H} A\right)=\rho\left(b_{g}\right)\left(e_{H} A\right) \rho\left(b_{g}\right)^{-1},
$$

and so $\rho\left(b_{g}\right)\left(e_{H} A\right)=\left(e_{H} A\right) \rho\left(b_{g}\right)$ for all $g \in H$. Then, by Schur's lemma, $e_{H} A=0$ if $M \not N$ and $e_{H} A$ is a constant if $M=N$.

Theorem A.13. Let $R^{\lambda}$ be a simple $R$-module and let $H$ be the inertia group of $R^{\lambda}$. The ring $R^{G}$ acts on $R^{\lambda}$ (by restriction) and $(\mathbb{C} H)_{\alpha}$ acts on $R^{\lambda}$ (by the $R$-module isomorphisms $\phi_{h}: R^{\lambda} \cong{ }^{h} R^{\lambda}$ of (A.3)) and these two actions commute. Thus there is a decomposition

$$
R^{\lambda} \cong \bigoplus_{\nu \in \hat{H}_{\alpha}} R^{\lambda, \nu} \otimes\left(H^{\nu}\right)^{*}
$$

where $\hat{H}_{\alpha}$ is an index set for the simple $(\mathbb{C} H)_{\alpha}$-modules, $\left(H^{\nu}\right)^{*}$ is the dual of the simple $(\mathbb{C} H)_{\alpha^{-1}-}$ module $H^{\nu}$, and $R^{\lambda, \nu}$ is an $R^{G}$-module. 
(a) If $R^{\lambda, \mu} \neq 0$ then it is a simple $R^{G}$-module.

(b) Every simple $R^{G}$-module is isomorphic to some $R^{\lambda, \mu}$.

(c) The nonzero $R^{\lambda, \mu}$ are pairwise nonisomorphic.

Proof. The setup of Lemma A.9(b) puts us in the situation of [Gr, §6.2]. If $e$ is the idempotent used in Lemma A.9 then the functor

$$
\begin{array}{ccc}
R \rtimes G \text {-modules } & \longrightarrow & R^{G} \text {-modules } \\
M & \longmapsto & e M
\end{array}
$$

is an exact functor such that if $M$ is a simple $R \rtimes G$-module then $e M$ is either 0 or a simple $R^{G_{-}}$ module. Furthermore, every simple $R^{G}$-module arises as $e M$ for some simple $R \rtimes G$-module $M$.

Let $R G^{\lambda, \mu}$ be a simple $R \rtimes G$-module as given by Theorem A.6. From the definition of $R G^{\lambda, \mu}$ we obtain

$$
\begin{aligned}
e R G^{\lambda, \mu} & =e(R \rtimes G) \otimes_{R \rtimes H}\left(R^{\lambda} \otimes H^{\mu}\right) \\
& =e \otimes\left(R^{\lambda} \otimes H^{\mu}\right)=e e_{H} \otimes\left(R^{\lambda} \otimes H^{\mu}\right) \\
& =e \otimes e_{H}\left(R^{\lambda} \otimes H^{\mu}\right),
\end{aligned}
$$

where $e_{H}=(1 /|H|) \sum_{h \in H} h$. Using the decomposition in the statement of the Theorem, we conclude that, as $R^{G}$-modules,

$$
\begin{aligned}
e R G^{\lambda, \mu} & =e \otimes e_{H}\left(\bigoplus_{\nu \in \hat{H}} R^{\lambda, \nu} \otimes\left(H^{\nu}\right)^{*} \otimes H^{\mu}\right) \\
& =e \otimes e_{H}\left(\bigoplus_{\nu \in \hat{H}} R^{\lambda, \nu} \otimes e_{H}\left(\left(H^{\nu}\right)^{*} \otimes H^{\mu}\right)\right) \\
& \cong R^{\lambda, \mu} .
\end{aligned}
$$

The last isomorphism is a consequence of Lemma A.12. The statement of the Theorem now follows from the results of J.A. Green quoted above.

Remark A.14. It follows from Theorem A.13 that $R^{\lambda}$ is semisimple as an $R^{G}$-module and the action of $(\mathbb{C} H)_{\alpha}$ on $R^{\lambda}$ generates $\operatorname{End}_{R^{G}}\left(R^{\lambda}\right)$. 


\section{REFERENCES}

[Ar1] S. ARIKI On the semisimplicity of the Hecke algebra of $(\mathbb{Z} / r \mathbb{Z})$ 乙 $S_{n}$, J. Algebra 169 (1994), $216-225$.

[Ar2] S. ARIKI Representation theory of a Hecke algebra of $G(r, p, n)$, J. Algebra 177 (1995), 164185.

[Ar3] S. ARIKI, On the decomposition numbers of the Hecke algebra of $G(m, 1, n)$, J. Math. Kyoto Univ. 36 (1996), 789-808.

[AK] S. ARIKI And K. KoIKe, A Hecke algebra of $(\mathbb{Z} / r \mathbb{Z}) \backslash S_{n}$ and construction of its irreducible representations, Adv. in Math. 106 (1994), 216-243.

[AM] S. ARIKI AND A. Mathas, On the number of simple modules of the Hecke algebras of type $G(r, p, n)$, Math. Zeitschrift 233 (2000), no. 3, 601-623.

[Bou1] N. Bourbaki, Groupes et algèbres de Lie, Chapitres 4,5 et 6, Elements de Mathématique, Hermann, Paris 1968.

[Bou2] N. Bourbaki, Algèbre, Chapitre 8, Elements de Mathématique, Hermann, Paris 1958.

[BMM] M. Broué, G. Malle, And J. Michel, Représentations unipotents génériques et blocs des groupes réductifs finis, Astérique 212, 1993.

[Ch1] I. Cherednik, A new interpretation of Gel'fand-Tzetlin bases, Duke Math. J. 54 (1987), $563-577$.

[Ch2] I. CheREDNIK, Monodromy representations for generalized Knizhnik-Zamolodchikov equations and Hecke algebras, Publ. RIMS, Kyoto Univ. 27 (1991), 711-726.

[Cr] J. CRISP, Ph.D. Thesis, University of Sydney, 1997.

[CR] C. Curtis and I. Reiner, Methods of representation theory-with applications to finite groups and orders Vol. I, J. Wiley and Sons, 1981.

[DJ] R. Dipper And G.D. JAmes, Blocks and idempotents of Hecke algebras of general linear groups, Proc. London Math. Soc. (3) 54 (1987) 57-82.

[DJM] R. Dipper, G.D. James And G.E. Murphy, Hecke algebras of type $B_{n}$ at roots of unity, Proc. London Math. Soc. (3) 70 (1995) 505-528.

[GL] M. Geck And S. Lambropoulou, Markov traces and knot invariants related to IwahoriHecke algebras of type B, J. Reine Angew. Math. 482 (1997), 191-213.

[Gr] J.A. Green, Polynomial representations of $G L_{n}$, Lecture Notes in Mathematics 830, SpringerVerlag, New York, 1980.

[Gy] A. Gyoja, A q-analogue of Young symmetrizer, Osaka J. Math. 23 (1986), 841-852.

[HR] T. Halverson And A. RAm, Murnaghan-Nakayama rules for characters of Iwahori-Hecke algebras of the complex reflection groups $G(r, p, n)$, Canadian J. Math. 50 (1998), 167-192.

[Hf] P.N. Hoefsmit, Representations of Hecke algebras of finite groups with BN-pairs of classical type, Ph.D. Thesis, University of British Columbia, 1974.

[IM] N. Iwahori And H. Matsumoto, On some Bruhat decomposition and the structure of the Hecke rings of p-adic Chevalley groups, Publ. Math. I.H.E.S. 25 (1965), 5-48. 
[Jo] V.F.R. Jones, A quotient of the affine Hecke algebra in the Brauer algebra, Enseign. Math. (2) 40 (1994), 313-344.

[KL] D. Kazhdan and G. Lusztig, Proof of the Deligne-Langlands conjecture for Hecke algebras, Invent. Math. 87 (1987), 153-215.

[Lu] G. LuszTig, Affine Hecke algebras and their graded version, J. Amer. Math. Soc. 2 (1989), 599-635.

[Mac] I.G. Macdonald, Symmetric functions and Hall polynomials, Second edition, Oxford University Press, New York, 1995.

[Mac2] I.G. Macdonald, Polynomial functors and wreath products, J. Pure and Appl. Algebra 18 (1980) 173-204.

[Mac3] I.G. MACDOnAld, Affine Hecke algebras and orthogonal polynomials, Séminaire Bourbaki, 47ème année, no 797, 1994-95, Astérisque 237 (1996), 189-207.

[Ra1] A. RAM, Seminormal representations of Weyl groups and Iwahori-Hecke algebras, Proc. London Math. Soc. (3) 75 (1997), 99-133.

[Ra2] A. RAM, Calibrated representations of affine Hecke algebras, preprint 1998, results to appear in J. Algebra under the title Affine Hecke algebras and generalized standard Young tableaux.

[Ra3] A. RAM, Standard Young tableaux for finite root systems, preprint 1998, results to appear in J. Algebra under the title Affine Hecke algebras and generalized standard Young tableaux.

[Ra4] A. RAM, Irreducible representations of rank two affine Hecke algebras, preprint 1998.

[Ra5] A. RAM, Skew shape representations are irreducible, preprint 1998.

[Re] M. REEDER, Isogenies of Hecke algebras and a Langlands correspondence for ramified principal series representations, Represent. Theory 6 (2002), 101-126.

[RR] A. RAm And J. RAmAgGe, Langlands parameters for affine Hecke algebra representations, in preparation.

[Sp] W. Specht, Eine Verallgemeinerung der symmetrischen Gruppe, Schriften Math. Seminar Berlin 1 (1932), 1-32.

[ST] G. C. Shephard And J.A. Todd, Finite unitary reflection groups, Canadian Journal of Math. 6 (1954), 274-304.

[Wz] H. Wenzl, Hecke algebras of type $A_{n}$ and subfactors, Invent. Math. 92 (1988), 349-383.

[Yg1] A. Young, On quantitative substitutional analysis (fifth paper), Proc. London Math. Soc. (2) 31 (1929), 273-288.

[Yg2] A. Young, On quantitative substitutional analysis (sixth paper), Proc. London Math. Soc. (2) 34 (1931), 196-230. 